\title{
FINITE RANK TRANSFORMATION AND WEAK CLOSURE THEOREM
}

\author{
Jan KWIATKOWSKI ${ }^{\mathrm{a}}$, Yves LACROIX ${ }^{\mathrm{b}}$ \\ ${ }^{a}$ Wydziat Matematyki i Informatyki, U. M. K., ul. Chopina 12/18, 87-100 Torun, Poland \\ ${ }^{\mathrm{b}}$ U.P.J.V., Lamfa, 33 rue St Leu, 80000 Amiens, France
}

Received 7 December 1999, revised 26 February 2001

En mémoire d'Anzelm Iwanik

ABSTRACT. - We introduce a new class of cocycles which provides examples of measure preserving dynamical systems $(X, \mathcal{B}, \mu, T)$, such that given positive integers $r \geqslant 2$ and $m \geqslant 1$, possibly infinite, with $(r, m) \neq(\infty, \infty)$, the rank is $r$ and the order of the quotient group in the measure-theoretic centralizer, $\# \frac{C(T)}{\operatorname{wcl}\left\{T^{n} ; n \in \mathbb{Z}\right\}}$, is $m$. Moreover, $\operatorname{wcl}\left\{T^{n} ; n \in \mathbb{Z}\right\}$ is uncountable. For the case $(r, m)=(\infty, \infty)$, we produce a mixing $T$. This completes the weak closure theorem of Jonathan King. ๑ 2002 Éditions scientifiques et médicales Elsevier SAS

AMS classification: 28D; 47A

Keywords: Rank; Measure-theoretic centralizer; Weak closure Theorem

RÉSUMÉ. - Nous introduisons une nouvelle classe de cocycles qui permet d'obtenir des exemples de flots $(X, \mathcal{B}, \mu, T)$, tels qu'étant donnés deux entiers $r \geqslant 2$ et $m \geqslant 1$, éventuellement infinis, avec $(r, m) \neq(\infty, \infty)$, le rang soit $r$ et l'ordre du groupe quotient dans le centralisateur, $\# \frac{C(T)}{\operatorname{wcl}\left\{T^{n} ; n \in \mathbb{Z}\right\}}$, soit $m$. En outre ces exemples sont tels que wcl $\left\{T^{n} ; n \in \mathbb{Z}\right\}$ est non dénombrable. Pour $(r, m)=(\infty, \infty)$, nous construisons un exemple avec $T$ mélangeant. Ceci en particulier complète le Théorème de Clôture Faible de Jonathan King. @ 2002 Éditions scientifiques et médicales Elsevier SAS

\section{Introduction}

Let $(X, \mathcal{B}, \mu, T)$ be an ergodic dynamical system and let $C(T)$ be the measuretheoretic centralizer of $T$. The Weak Closure Theorem [10] asserts that $C(T)$ coincides with the weak closure of the set of powers of $T$, denoted $\operatorname{wcl}\left\{T^{n}, n \in \mathbb{Z}\right\}$, whenever $r(T)=1$, where $r(T)$ is the rank of $T$.

Hence the question of the existence of a relationship between $r(T)$ and the cardinality $q(T)$ of the quotient group $\frac{C(T)}{\operatorname{wcl}\left\{T^{n} ; n \in \mathbb{Z}\right\}}$ in the general case naturally arises.

E-mail addresses: jkwiat@mat.uni.torun.pl (J. Kwiatkowski), yves.lacroix@u-picardie.fr (Y. Lacroix). 
For mixing $T$ it follows from [11] that $q(T) \leqslant r(T)$. It is not difficult to show that the same inequality holds for $T$ 's defined in [2] - generalized Morse flows, for which $q(T)=2$ and $r(T)$ can be arbitrarily large.

Each automorphism $T$ in [2] has a partially discrete spectrum whence the two kinds of automorphisms previously described are rather far from each other.

However for both cases $\operatorname{wcl}\left\{T^{n}, n \in \mathbb{Z}\right\}=\left\{T^{n}, n \in Z\right\}$. Therefore an interesting additional feature is to construct arbitrary pairs $(q(T), r(T))$ with an uncountable $\operatorname{wcl}\left\{T^{n}, n \in Z\right\}$.

In this paper we shall introduce some new classes of cocycles, which define flows that are ergodic group extensions of rank 1 systems, and are tractable enough to allow an exact computation of both $r(T)$ and $q(T)$.

We shall pick within these classes, for each possible pair $(q, r)$, examples of ergodic automorphisms $T$ such that $(q(T), r(T))=(q, r)$. Moreover, for $(q, r) \neq(\infty, \infty)$, our examples produce an uncountable $\operatorname{wcl}\left\{T^{n}, n \in Z\right\}$.

The difficulty lies both in the proposition of a good candidate, and in the computation of the rank and the order of the quotient group. For $(r, m) \neq(\infty, \infty)$, our examples lie in the class of group extensions determined by $r$-Toeplitz sequences. For the $(\infty, \infty)$ case, the example is mixing: it is a weakly mixing extension of a rank 1 mixing transformation [1].

From these examples, it now follows that in its generality, the weak closure theorem is the only one for limitations concerning the coexistence of the measure-theoretic invariants $q(T)$ and $r(T)$.

The investigations of ergodicity and that of the measure-theoretic centralizer both rely on Newton's functional equation [21] and are carried out partially on a measure-theoretic group extension representation of the system.

Investigating the rank (and partly the centralizer too) we use a shift representation of those extensions.

\section{Preliminaries}

\subsection{Notations and definitions}

Let $(X, \mathcal{B}, \mu)$ be a Lebesgue space and $T$ a measure-preserving invertible ergodic transformation of $(X, \mathcal{B}, \mu)$. By the centralizer (measure-theoretic) of $T$ we mean the set of all measure-preserving automorphisms of $(X, \mathcal{B}, \mu)$ which commute with $T$ and we denote it by $C(T)$. Then $C(T)$ is a topological group with the standard operation of composition of transformations and with a topology (called the weak topology) defined as follows: $\left\{S_{n}\right\}_{n \in \mathbb{N}} \in C(T)$ converges to $S \in C(T)$ if for every $A \in \mathcal{B}$

$$
\mu\left(S_{n} A \triangle S A\right) \longrightarrow 0 .
$$

We shall indicate this convergence by $S_{n} \rightarrow S$. With this topology, $C(T)$ is metric, complete. By wcl $\left\{T^{n}, n \in \mathbb{Z}\right\}$ we mean the weak closure of the powers of $T$ in $C(T)$.

We say that a sequence of sets $A_{1}, \ldots, A_{k} \in \mathcal{B}$ is a $T$-stack if these sets are pairwise disjoint and $T A_{i}=A_{i+1}, i=1, \ldots, k-1$. 
If we are given a collection of $r$ measurable subsets $F_{i}$ of $X$, and $r$ positive integers $h_{i}$, such that $\mathcal{C}:=\left\{T^{l} F_{i}: 1 \leqslant i \leqslant r, 0 \leqslant l<h_{i}\right\}$ is a collection of disjoint sets (a union of $r$ disjoint $T$-stacks), setting $Y=X \backslash\left(\bigcup_{C \in \mathcal{C}} C\right)$, this union of $r$ disjoint $T$-stacks defines a partition $\tilde{\mathcal{C}}:=\mathcal{C} \cup\{Y\}$, and a $\sigma$-algebra $\sigma(\tilde{\mathcal{C}})$.

The rank of $T$ is the smallest integer $r$ such that given $\varepsilon>0$, there exists a union of $r$ disjoint $T$-stacks $\mathcal{C}$, such that for any measurable $A \in \mathcal{B}$, there exists $B \in \sigma(\tilde{\mathcal{C}})$ with $\mu(A \Delta B)<\varepsilon$. If such a positive integer does not exist then we say that $r(T)=\infty$.

We shall give a symbolic version for the definition of the rank in 2.2. and 4., which are shown to be equivalent to the one above in [3] and [20]. We reffer the interested reader to [5-7] for more on rank and partitions.

Suppose now that $G$ is a compact metric abelian group and $\varphi: X \longrightarrow G$ is a measurable function which we will call a cocycle. The $G$-extension of $(X, \mathcal{B}, \mu, T)$ given by the cocycle $\varphi$ is the dynamical system $\mathcal{X}_{\varphi}=\left(X \times G, \mathcal{B} \times \mathcal{B}_{G}, \mu \times \nu, T_{\varphi}\right)$, where $\mathcal{B}_{G}$ is the Borel $\sigma$-algebra in $G, v$ is the normalized Haar measure on $G$ and

$$
T_{\varphi}(x, g)=(T x, g+\varphi(x))
$$

for $x \in X, g \in G$. It is well known [22] that for ergodic $(X, \mathcal{B}, \mu, T)$ the following theorem is true.

THEOREM A. $-T_{\varphi}$ is ergodic iff the functional equation

$$
\frac{f(T x)}{f(x)}=\gamma(\varphi(x))
$$

has no measurable solutions $f: X \longrightarrow K$ for any nontrivial character $\gamma$ of $G$ ( $K$ is the unit complex circle).

It is known (see [21] for the definition) that if $(X, \mathcal{B}, \mu, T)$ is a canonical factor of $T_{\varphi}$ (for example if $T$ is with discrete spectrum) then, assuming that $T_{\varphi}$ is ergodic, $C\left(T_{\varphi}\right)$ is given by the triples $(S, f, \tau)$, where $S \in C(T), f: X \rightarrow G$ is measurable and $\tau$ is a group automorphism of $G$ such that

$$
f(T x)-f(x)=\varphi(S x)-\tau(\varphi(x)) .
$$

This means that every element $R \in C\left(T_{\varphi}\right)$ is of a form

$$
R(x, g)=(S x, \tau(g)+f(x)) .
$$

In such a case we write $R \sim(S, f, \tau)$. The following property is proved in [17] and [18], using Theorem A.

THEOREM B. - If $R_{n}, R \in C\left(T_{\varphi}\right)$ and $R_{n} \sim\left(S_{n}, f_{n}\right.$, id $), R \sim\left(S, f\right.$, id) then $R_{n} \rightarrow R$ iff $S_{n} \rightarrow S$ and $f_{n} \longrightarrow f$ in measure $\mu$.

Let $\sigma_{a}: X \times G \longrightarrow X \times G$ be given by the formula

$$
\sigma_{a}(x, g)=(x, g+a), \quad a \in G .
$$


Then $\sigma_{a} \in C\left(T_{\varphi}\right), \sigma_{a} \sim(\mathrm{id}, a, \mathrm{id})$. For every integer $n,\left(T_{\varphi}\right)^{n}$ is given by the formula

$$
\left(T_{\varphi}\right)^{n}(x, g)=\left(T^{n} x, g+\varphi^{(n)}(x)\right),
$$

where

$$
\varphi^{(n)}(x)= \begin{cases}\varphi(x)+\cdots+\varphi\left(T^{n-1} x\right), & \text { if } n \geqslant 0, \\ -\varphi\left(T^{-1} x\right)-\cdots-\varphi\left(T^{n} x\right), & \text { if } n<0 .\end{cases}
$$

Then it follows from Theorem B that

COROLlary 1. $-\left(T_{\varphi}\right)^{n_{k}} \rightarrow \sigma_{a}$ in $C\left(T_{\varphi}\right)$ iff $T^{n_{k}} \rightarrow$ id in $C(T)$ and $\varphi^{\left(n_{k}\right)} \longrightarrow$ a in measure.

\subsection{Sequences and blocks}

A finite sequence $B=(B[0], \ldots, B[k-1]), B[i] \in G, 0 \leqslant i \leqslant k-1, k \geqslant 1$, is called a block over $G$. The number $k$ is called the length of $B$ and is denoted by $|B|$. If $C=(C[0], \ldots, C[n-1])$ is another block then the concatenation of $B$ and $C$ is the block

$$
B C=(B[0], \ldots, B[k-1], C[0], \ldots, C[n-1]) .
$$

Inductively we define the concatenation of an arbitrary number of blocks. By $B_{g}, g \in G$, we will denote the block

$$
B_{g}=(B[0]+g, \ldots, B[k-1]+g)
$$

and by $B[i, s](0 \leqslant i \leqslant s \leqslant k-1)$ the block

$$
B[i, s]=(B[i], \ldots, B[s]) .
$$

Assume that

$$
B=B(0) \ldots B(r-1)
$$

is a concatenation of $r$ blocks $B(0), \ldots, B(r-1)$ having the same lengths and

$$
C=C[0] \ldots C[r m-1]
$$

for some $m \geqslant 1$. We define the product $B \stackrel{r}{\times} C$ of $B$ and $C$ as follows:

$$
\begin{aligned}
B \stackrel{r}{\times} C= & B_{C[0]}(0) \ldots B_{C[r-1]}(r-1) B_{C[r]}(0) \ldots \\
& B_{C[2 r-1]}(r-1) B_{C[r(m-1)]}(0) \ldots B_{C[r m-1]}(r-1) .
\end{aligned}
$$

Then

$$
|B \stackrel{r}{\times} C|=\frac{|B||C|}{r}=|B(i)| r m, \quad \text { for every } i=0, \ldots, r-1 .
$$

Let $\Omega$ by the space of all bi-infinite sequences over $G$. If $\omega \in \Omega$ or $\omega$ is a onesided infinite sequence over $G$ then $\omega[i, s], i \leqslant s$, denotes the block $(\omega[i], \ldots, \omega[s])$. 
A block $B$ is said to occur at place $i$ in $\omega$ (resp. in a block $C,|C|=n$, if $|B| \leqslant n$ ) if $\omega[i, i+|B|-1]=B$ (resp. $C[i, i+|B|-1]=B$ ). The frequencies of $B$ in $C$ or $\omega$ are the numbers

$$
\begin{gathered}
\operatorname{fr}(B, C)=|C|^{-1} \#\{0 \leqslant i \leqslant|C|-|B| ; B \text { occurs at place } i \text { in } C\}, \\
\operatorname{fr}(B, \omega)=\lim _{s \rightarrow \infty} \operatorname{fr}(B, \omega[0, s-1]),
\end{gathered}
$$

if this limit exists.

For an infinite subsequence of $\omega, E=\{\omega[n], n \in I \subset \mathbb{Z}\}$ (resp. $E=\{\omega[n], n \in I \subset$ $\mathbb{N}\}$ ), we call the density of $E$ the density of the set $I$ in $\mathbb{Z}$ (resp. in $\mathbb{N}$ ), whenever it exists. Let $\delta>0$. We say that $B \delta$-occurs at place $i$ in $C$ (resp. in $\omega$ ) if

$$
d(B, C[i, i+|B|-1])<\delta \quad(\operatorname{resp} . d(B, \omega[i, i+|B|-1])<\delta),
$$

where

$$
d\left(\left(x_{1}, \ldots, x_{n}\right),\left(y_{1}, \ldots, y_{n}\right)\right)=n^{-1} \#\left\{i ; x_{i} \neq y_{i}\right\}
$$

( $d$ is called the normalized Hamming distance or $d$-bar distance between sequences). We will say also that $B \delta$-occurs on the fragment $\omega[i, i+|B|-1]$ of $\omega$.

We will use the following elementary properties of the distance $d$;

$$
\begin{gathered}
d(B \stackrel{r}{\times} C, B \stackrel{r}{\times} D)=d(C, D) \quad(\text { see }(7)), \\
d\left(B_{g}, C_{g}\right)=d(B, C), \\
d\left(A_{1} A_{2}, B_{1} B_{2}\right)=\frac{\left|A_{1}\right|}{\left|A_{1}\right|+\left|A_{2}\right|} d\left(A_{1}, B_{1}\right)+\frac{\left|A_{2}\right|}{\left|A_{1}\right|+\left|A_{2}\right|} d\left(A_{2}, B_{2}\right),
\end{gathered}
$$

where $\left|A_{1}\right|=\left|B_{1}\right|,\left|A_{2}\right|=\left|B_{2}\right|$.

If $D_{1} \subset D\left(D_{1}\right.$ is a subblock of $\left.D\right)$ and $C_{1} \subset C,\left|D_{1}\right|=\left|C_{1}\right|$, both appearing in the corresponding same positions, then

$$
\begin{gathered}
d(D, C) \geqslant \frac{\left|D_{1}\right|}{|D|} d\left(D_{1}, C_{1}\right) . \\
d\left(A_{1} A_{2} \ldots A_{s}, B_{1} B_{2} \ldots B_{s}\right)=\frac{1}{s} \sum_{i=1}^{s} d\left(A_{i}, B_{i}\right)
\end{gathered}
$$

if $\left|A_{1}\right|=\left|A_{2}\right|=\cdots=\left|A_{s}\right|=\left|B_{1}\right|=\cdots=\left|B_{s}\right|$.

By $T_{\sigma}$ we denote the left shift homeomorphism of $\Omega$. If $\omega \in \Omega$ then $O(\omega)$ denotes the $T_{\sigma}$-orbit of $\omega$ and $\Omega_{\omega}$ the $T_{\sigma}$-orbit closure of $\omega$ in $\Omega$. The $T_{\sigma}$-orbit closure $\Omega_{\omega}$ is well-defined if $\omega$ is a one-sided sequence. Namely, we first let $\diamond \notin G$ be an additional symbol. Then we let $\omega^{\diamond}$ denote the bi-infinite sequence which agrees with $\omega$ at positive coordinates and has only squares appearing at the negative ones. Then we say that a bi-infinite $y$ belongs to $\Omega_{\omega}$ if there exists $n_{i} \rightarrow+\infty$ such that $T_{\sigma}^{n_{i}} \omega \rightarrow y$ in $\Omega$ (the 
convergence is for all coordinates of $y$, and the limiting element $y$ does not contain any more squares). The topological flow $\left(\Omega_{\omega}, T_{\sigma}\right)$ is called minimal if there is no non trivial closed and $T_{\sigma}$-invariant subset of $\Omega_{\omega}$. We say that $\left(\Omega_{\omega}, T_{\sigma}\right)$ is uniquely ergodic if there is a unique borelian normalized $T_{\sigma}$-invariant measure $\mu_{\omega}$ on $\Omega_{\omega}$. Then $\left(\Omega_{\omega}, T_{\sigma}\right)$ is said to be strictly ergodic if it is minimal and uniquely ergodic. Suppose $\left(\Omega_{\omega}, T_{\sigma}\right)$ is strictly ergodic. The unique $T_{\sigma}$-invariant measure $\mu_{\omega}$ is determined by the condition

$$
\mu_{\omega}(B)=\operatorname{fr}(B, \omega)
$$

for each block $B$. In the case of a discrete group $G$, the definition of the rank has the following symbolic transcription.

The system $\left(\Omega_{\omega}, T_{\sigma}, \mu_{\omega}\right)$ is of rank at most $r$ if for any $\delta>0$ and every $n$, there exist $r$ blocks $B_{1}, \ldots, B_{r},\left|B_{i}\right| \geqslant n$, such that for all $N$ large enough, for any $s \in \mathbb{N}$, the fragment $\omega[s, s+N-1]$ has a form

$$
\omega[s, s+N-1]=\varepsilon_{1} W_{1} \varepsilon_{2} W_{2} \cdots \varepsilon_{k} W_{k} \varepsilon_{k+1},
$$

where $\left|\varepsilon_{1}\right|+\cdots+\left|\varepsilon_{k}\right|+\left|\varepsilon_{k+1}\right|<\delta N$ and the distance $d$ between $W_{j}$ and some $B_{m}, j=1, \ldots, k, 1 \leqslant m \leqslant r$, is less than $\delta$. The system $\left(\Omega_{\omega}, T_{\sigma}, \mu_{\omega}\right)$ is of rank $r$ if it is of rank at most $r$ and not of rank at most $r-1$.

\subsection{Adding machines and $r$-Toeplitz cocycles}

Now, let $T:(X, \mathcal{B}, \mu) \longrightarrow(X, \mathcal{B}, \mu)$ be a $\left\{p_{t}\right\}$-adic adding machine i.e.

$$
\begin{gathered}
p_{t+1}=\lambda_{t+1} p_{t}, \quad \lambda_{0}=p_{0}, \quad \lambda_{t} \geqslant 2 \text { for } t \geqslant 0, \\
X=\left\{x=\sum_{t=0}^{\infty} q_{t} p_{t-1} ; 0 \leqslant q_{t} \leqslant \lambda_{t}-1, p_{-1}=1\right\}
\end{gathered}
$$

is the group of $\left\{p_{t}\right\}$-adic integers and $T x=x+\hat{1}$, where

$$
\hat{1}=1+0 p_{1}+0 p_{2}+\cdots .
$$

The space $X$ has a standard sequence $\left\{\xi_{t}\right\}_{t \geqslant 0}$ of $T$-stacks. Namely

$$
\xi_{t}=\left(D_{0}^{t}, \ldots, D_{p_{t}-1}^{t}\right),
$$

where

$$
D_{0}^{t}=\left\{x \in X ; q_{0}=\cdots=q_{t}=0\right\}, \quad D_{s}^{t}=T^{s}\left(D_{0}^{t}\right)
$$

for $s=1, \ldots, p_{t}-1$. We have

$$
X=\bigcup_{i=0}^{p_{t}-1} D_{i}^{t} .
$$

Then $\xi_{t+1}$ refines $\xi_{t}$ and the sequence of partitions $\left\{\xi_{t}\right\}_{t \geqslant 0}$ converges to the point partition. 
We will define a special class of cocycles $\varphi: X \longrightarrow G$ that are determined by Toeplitz sequences over $G$.

Let $r \geqslant 2$ be an integer, and assume that $b^{0}, b^{1}, \ldots$ are finite blocks over $G$ with $\left|b^{t}\right|=\lambda_{t} r, \lambda_{t} \geqslant 2$, such that

$$
b^{t}[0, r-1]=(\underbrace{0, \ldots, 0}_{r \text { times }}) .
$$

We shall introduce a particular sequence $\left(p_{t}\right)$, and some new blocks $\left(B^{t}\right)$.

We can write

$$
b^{t}=b^{t}(0) \ldots b^{t}(r-1), \quad\left|b^{t}(i)\right|=\lambda_{t}, i=0, \ldots, r-1 .
$$

Define another sequence of blocks $\left\{B^{t}\right\}$ letting

$$
B^{0}=b^{0}, \quad B^{t+1}=B^{t} \stackrel{r}{\times} b^{t+1}, \quad t \geqslant 0 .
$$

Then we have

$$
\left|B^{t}\right|=r m_{t}=p_{t}, \quad m_{t}=\lambda_{0} \cdots \lambda_{t},
$$

and we can represent $B^{t}$ as

$$
B^{t}=B^{t}(0) \cdots B^{t}(r-1), \quad\left|B^{t}(i)\right|=m_{t}, i=0, \ldots, r-1 .
$$

Moreover

$$
B^{t+1}\left[0, p_{t}-1\right]=B^{t}
$$

Now we can define a cocycle $\varphi$ by

$$
\varphi(x)=B^{t}[i+1]-B^{t}[i]
$$

if $x \in D_{i}^{t}$ except of $i=m_{t}-1,2 m_{t}-1, \ldots, p_{t}-1$. Let us observe that $\varphi$ is well defined. Such a cocycle is called $r$-Toeplitz cocycle. For every $t \geqslant 0, \varphi$ is constant on the levels of $\xi_{t}$ except of $r$ levels.

The sequence $\left\{B^{t}\right\}_{t \geqslant 0}$ determines a one-sided sequence $\omega$ as follows:

$$
\omega\left[0, p_{t}-1\right]=B^{t}, \quad t=0,1, \ldots
$$

The condition (17) guarantees that $\omega$ is well defined.

It is not hard to show that the condition

$$
\operatorname{fr}\left(g, b^{t}\right) \geqslant \rho>0 \quad \text { (if } G \text { is finite) }
$$

for every $g \in G$ and $t=0,1 \ldots$, implies that the system $\left(\Omega_{\omega}, T_{\sigma}\right)$ is strictly ergodic. Then using (19), (20), and arguments as in [16], we deduce that the dynamical systems $\left(\Omega_{\omega}, T_{\sigma}, \mu_{\omega}\right)$ and $\left(X \times G, T_{\varphi}, \mu \times v\right)$ are measure-theoretically isomorphic when $T_{\varphi}$ is ergodic. 
The group extensions defined by $r$-Toeplitz cocycles shall be called $r$-Toeplitz extensions.

In the sequel we will write

$$
\omega=b^{0} \stackrel{r}{\times} b^{1} \stackrel{r}{\times} b^{2} \stackrel{r}{\times} \cdots .
$$

Except of $\omega$ we need the sequences $\omega_{t}, t \geqslant 0$, defined by

$$
\omega_{t}=b^{t} \stackrel{r}{\times} b^{t+1} \stackrel{r}{\times} \cdots
$$

\section{Examples of $r$-Toeplitz extensions}

In this part, given $r \geqslant 2$ and $m \geqslant 1$, we define $r$-Toeplitz group extensions having cardinality of the quotient group $C\left(T_{\varphi}\right) / \operatorname{wcl}\left\{T_{\varphi}^{n} ; n \in \mathbb{Z}\right\}$ equal to $m$.

\subsection{The case $r \geqslant 2, m \geqslant 2$}

Let $G=\mathbb{Z} / m \mathbb{Z}=\{0, \ldots, m-1\}$. Define

$$
\begin{aligned}
F^{(i)} & =\overbrace{00 \ldots 0}^{r\left(2^{i+2}-1\right)} \overbrace{0 \ldots 0 \underbrace{1}_{i+1} 0 \ldots 0}^{r}, \quad i=0, \ldots, r-1 ; \\
H^{(i)} & =F_{0}^{(i)} F_{1}^{(i)} \ldots F_{m-1}^{(i)} .
\end{aligned}
$$

We have $\left|H^{(i)}\right|=m r 2^{i+2}$. Next define

$$
\begin{aligned}
b^{t}(0)= & \underbrace{H^{(0)} H^{(0)} \ldots H^{(0)}}_{x_{0} \text { times }} \\
b^{t}(1)= & \underbrace{H^{(1)} H^{(1)} \ldots H^{(1)}}_{x_{1} \text { times }} \\
b^{t}(r-1)= & \underbrace{H^{(r-1)} H^{(r-1)} \ldots H^{(r-1)}}_{x_{r-1} \text { times }}
\end{aligned}
$$

where

$$
x_{i}=2^{t+r-1-i}, \quad 0 \leqslant i \leqslant r-1,
$$

and

$$
b^{t}=b^{t}(0) \ldots b^{t}(r-1), \quad t \geqslant 0 .
$$

Then we have

$$
\lambda_{t}=\left|b^{t}(i)\right|=m r 2^{t+r+1}, \quad \text { for } i=0, \ldots, r-1 \quad \text { (see (13)) }
$$

and

$$
\left|b^{t}\right|=m r^{2} 2^{t+r+1} .
$$


Now define the blocks $B^{t}, t \geqslant 0$, by (14) and the cocycle $\varphi$ by (18). Then from (15)

$$
p_{t}=\left|B^{t}\right|=m^{t+1} r^{2 t} 2^{r+1}\left(2^{t+1}-1\right), \quad t \geqslant 0 .
$$

\subsection{The case $r \geqslant 2, m=1$}

Let $G=\mathbb{Z} / n \mathbb{Z}=\{0, \ldots, n-1\}, n \geqslant 4$. Then define

$$
\begin{aligned}
F^{(i)} & =\overbrace{00 \ldots 0}^{3 r} \overbrace{0 \ldots \underbrace{1}_{i+1} 0 \ldots 0}^{r} 0, \\
H^{(i)} & =F_{0}^{(i)} F_{1}^{(i)} \ldots F_{n-1}^{(i)},
\end{aligned}
$$

and

$$
b^{t}(i)=\underbrace{H^{(i)} H^{(i)} \ldots H^{(i)}}_{x \text { times }}, \quad x=2^{t} .
$$

Next set

$$
\begin{aligned}
& b^{t}=b^{t}(0) \ldots b^{t}(r-1), \\
& B^{t}=b^{0} \stackrel{r}{\times} b^{1} \stackrel{r}{\times} \cdots \stackrel{r}{\times} b^{t}, \quad t \geqslant 0,
\end{aligned}
$$

and define $\varphi$ by (18). In this case we have

$$
\lambda_{t}=r n 2^{t+2}=\left|b^{t}(i)\right|, \quad\left|b^{t}\right|=r^{2} n 2^{t+2}, \text { for } i=0,1, \ldots, r-1 \text { and } t \geqslant 0 .
$$

\subsection{Ergodicity and the measure-theoretic centralizer}

THEOREM 1. $-T_{\varphi}$ is ergodic.

Proof. - We will prove ergodicity of $T_{\varphi}$ in both cases 3.1 and 3.2. Assume that there exists a measurable function $f: X \longrightarrow K$ satisfying (1). Then (see (5), (6))

$$
\frac{f\left(T^{n} x\right)}{f(x)}=\gamma\left(\varphi^{(n)}(x)\right)
$$

for $\mu$-a.e. $x \in X$ and every $n \in \mathbb{Z}$.

In particular (22) holds for $n=p_{t}, t=0,1, \ldots$ The measurability of $f$ and the fact that $\xi_{t} \longrightarrow \varepsilon$ (the partition into points) in $X$ imply

$$
\gamma\left(\varphi^{\left(p_{t}\right)}(x)\right)=1
$$

except of a subset of measure $\varepsilon_{t}$ and $\varepsilon_{t} \longrightarrow 0$.

Let $x \in D_{j}^{t+1}, 0 \leqslant j \leqslant p_{t+1}-1$. We can represent $j$ as

$$
j=u p_{t}+v m_{t}+\rho,
$$

where $0 \leqslant u \leqslant \lambda_{t+1}-1,0 \leqslant v \leqslant r-1,0 \leqslant \rho \leqslant m_{t}-1$ (see (15)). 
It follows from (18) (with $t:=t+1$ ) that

$$
\varphi^{\left(p_{t}\right)}(x)=B^{t+1}\left[j+p_{t}\right]-B^{t+1}[j]
$$

except $j$ for which $u=u_{1}=\frac{\lambda}{r}-1, \ldots, u=u_{r}=\frac{r \lambda}{r}-1=\lambda-1, \lambda=\lambda_{t+1}$. At the same time we have

$$
B^{t+1}[j]=b[u r+v]+B^{t}(v)[\rho], \quad b=b^{t+1} \quad(\text { see }(14),(16)) .
$$

Then (25) can be rewritten as

$$
\varphi^{\left(p_{t}\right)}(x)=b[(u+1) r+v]-b[u r+v], \quad u \neq u_{1}, \ldots, u_{r} .
$$

The last equality and (23) imply that

$$
\gamma(c[q])=1 \quad(q=u r+v)
$$

for $q \in V_{t} \subset\left\{0,1, \ldots, r \lambda_{t+1}-1\right\}, \frac{\# V_{t}}{r \lambda_{t+1}} \geqslant 1-\varepsilon_{t}-\frac{2}{\lambda_{t+1}}$, where $c=c^{t}$ is given by

$$
c[q]:=b[q+r]-b[q], \quad q=0, \ldots, r \lambda-r-1 .
$$

Further the blocks $c=c^{t}$ have the following forms:

$$
c=\overbrace{E^{(0)} \ldots E^{(0)}}^{\left(m x_{0}-1\right) \text { times }} L^{(0)} \overbrace{E^{(1)} \ldots E^{(1)}}^{\left(m x_{1}-1\right) \text { times }} L^{(1)} \ldots \overbrace{E^{(r-1)} \ldots E^{(r-1)}}^{\left(m x_{r-1}-1\right) \text { times }}
$$

where

$$
\begin{aligned}
E^{(0)}= & \overbrace{0 \ldots 0}^{2 r} \overbrace{10 \ldots 0}^{r} \overbrace{01 \ldots 1}^{r}, \quad\left|L^{0}\right|=r, \\
E^{(1)}= & \overbrace{0 \ldots 0}^{6 r} \overbrace{010 \ldots 0}^{r} \overbrace{101 \ldots 1}^{r}, \quad\left|L^{1}\right|=r, \\
& \vdots \\
E^{(r-1)}= & \overbrace{0 \ldots 0}^{r} \overbrace{0 \ldots 01}^{r+1} \overbrace{1 \ldots 10}^{r}, \quad\left|L^{(r-2)}\right|=r,
\end{aligned}
$$

in the case 3.1. In the case 3.2 we have

$$
c=\overbrace{E^{(0)} \ldots E^{(0)}}^{(n x-1) \text { times }} L^{(0)} \overbrace{E^{(1)} \ldots E^{(1)}}^{(n x-1) \text { times }} L^{(1)} \ldots \overbrace{E^{(r-1)} \ldots E^{(r-1)}}^{(n x-1) \text { times }}
$$

where

$$
\begin{aligned}
& E^{(0)}=\overbrace{0 \ldots 0}^{2 r} \overbrace{10 \ldots 0}^{r} \overbrace{01 \ldots 1}^{r}, \quad\left|L^{0}\right|=r, \\
& E^{(1)}=\overbrace{0 \ldots 0}^{2 r} \overbrace{010 \ldots 0}^{r} \overbrace{101 \ldots 1}^{r}, \quad\left|L^{1}\right|=r,
\end{aligned}
$$




$$
E^{(r-1)}=\overbrace{0 \ldots 0}^{2 r} \overbrace{0 \ldots 01}^{r} \overbrace{1 \ldots 10}^{r}, \quad\left|L^{(r-2)}\right|=r .
$$

In both cases 1 appears in $c$ with frequency $>\frac{1}{r 2^{r+2}}$ for each $t \geqslant 0$. Then (27) implies $\gamma(1)=1$ so $\gamma$ is trivial. We have proved that $T_{\varphi}$ is ergodic.

\subsection{The centralizer of $T_{\varphi}$}

The $p_{t}$-adic adding machine $(X, \mathcal{B}, \mu, T)$ is a canonical factor of the group extension $\left(X \times G, \mathcal{B} \times \mathcal{B}_{G}, \mu \times v, T_{\varphi}\right)$. Then $C\left(T_{\varphi}\right)$ is described in 2.1. We can distinguish the following subgroups of $C\left(T_{\varphi}\right)$ :

$$
\begin{aligned}
& C_{1}=\operatorname{wcl}\left\{T_{\varphi}^{n} ; n \in \mathbb{Z}\right\}, \\
& C_{2}=\left\{\sigma_{a} \circ \widetilde{S} ; \tilde{S} \in C_{1} \text { and } a \in G\right\}, \\
& C_{3}=\{R \sim(S, f, \tau) ; \tau=\mathrm{id}\} .
\end{aligned}
$$

Of course $C_{1}, C_{2}, C_{3}$ are closed subgroups of $C\left(T_{\varphi}\right)$ and

$$
C_{1} \subset C_{2} \subset C_{3} \subset C\left(T_{\varphi}\right) .
$$

We prove in Lemmas 1 and 2 that $C\left(T_{\varphi}\right)$ reduces to $C_{2}$ when $\varphi$ is the $r$-Toeplitz cocycle defined in 3.1 or in 3.2 .

In the sequel $n$ means the same $n$ as the one defined in 3.2 if this case is considered, and $n:=m$ if the case 3.1 is considered.

Lemma 1. $-C\left(T_{\varphi}\right)=C_{3}$.

Proof. - Take $R$ as in (3). Then the triple $(S, f, \tau)$ satisfies (2). Putting $x:=$ $T x, \ldots, T^{p_{t}-1} x$ in (2) and summing we obtain

$$
f\left(T^{p_{t}} x\right)-f(x)=\varphi^{\left(p_{t}\right)}(S x)-\tau\left(\varphi^{\left(p_{t}\right)}(x)\right)
$$

for $\mu$-a.e. $x \in X$ and each $t \geqslant 0$. Using the same arguments as in the proof of Theorem 1 we get from (30)

$$
\varphi^{\left(p_{t}\right)}(S x)-\tau\left(\varphi^{\left(p_{t}\right)}(x)\right)=0
$$

for $x \in X_{t}$ and $\mu\left(X_{t}\right) \longrightarrow 1$.

Further we know [21] that there exists $q_{0} \in X$ such that

$$
S(x)=x+g_{0}, \quad x \in X .
$$

Let

$$
g_{0}=\sum_{t=0}^{\infty} u_{t} p_{t-1}, \quad 0 \leqslant u_{t} \leqslant \lambda_{t}-1, t \geqslant 1 \text { and } 0 \leqslant u_{0} \leqslant \lambda_{0} r-1
$$


Fix $t$ and consider (31) on the stack $\xi_{t+1}$. Let

$$
j_{t}=\sum_{j=0}^{t} u_{j} p_{j-1}
$$

Then (see (24))

$$
j_{t}=v_{0} m_{t}+\rho_{0}, \quad j_{t+1}=u_{0} p_{t}+v_{0} m_{t}+\rho_{0}, \quad u_{0}=u_{t+1} .
$$

If $x \in D_{j}^{t+1}, 0 \leqslant j \leqslant p_{t+1}-1$, then $S x \in D_{j+j_{t+1}}^{t+1}$, where $j+j_{t+1}$ is taken $\bmod p_{t+1}$. We can write

$$
j+j_{t+1}=\bar{u} p_{t}+\bar{v} m_{t}+\bar{\rho}, \quad 0 \leqslant \bar{u} \leqslant \lambda-1,0 \leqslant \bar{v} \leqslant r-1,0 \leqslant \bar{\rho} \leqslant m_{t}-1 .
$$

Let us denote (use (24) for $j$ )

$$
q_{0}= \begin{cases}u_{0} r+v_{0} & \text { if } \rho=0, \ldots, m_{t}-\rho_{0}-1 \\ u_{0} r+v_{0}+1 & \text { if } \rho=m_{t}-\rho_{0}, \ldots, m_{t}-1\end{cases}
$$

and $q=u r+v, \bar{q}=\bar{u} r+\bar{v}$. Then $\bar{q}=q+q_{0}\left(\bmod r \lambda_{t+1}\right)$. Thus (26) and (31) give

$$
c\left[q+q_{0}\right]=\tau(c[q]) \quad \text { if } q \in V_{t} \subset\left\{0,1, \ldots, r \lambda_{t+1}-1\right\}
$$

and $\frac{1}{\lambda_{t+1}} \# V_{t} \longrightarrow 1$. Analysing the sequences (28) and (29) it is easy to observe that they do not satisfy (32) with any $q_{0}$ whenever $\tau \neq$ id (i.e., $\tau(1) \neq 1$ ). The lemma is proved.

LEMMA 2. $-C\left(T_{\varphi}\right)=C_{2}$.

Proof. - Let $R \sim(S, f$, id $) \in C_{3}$. Then (32) means

$$
c\left[q+q_{0}\right]=c[q], \quad q \in V_{t} .
$$

The last condition implies

$$
q_{0}(t)=q_{0}=2^{r+1} r m w, \quad w=w_{t},
$$

in the case 3.1 and

$$
q_{0}(t)=q_{0}=4 r n w, \quad w=w_{t},
$$

in the case 3.2, where $0 \leqslant w \leqslant r 2^{t+1}-1$ (see again (28) and (29)). Moreover

$$
\min \left(\frac{q_{0}(t)}{\lambda_{t+1}}, 1-\frac{q_{0}(t)}{\lambda_{t+1}}\right) \longrightarrow 0 .
$$

The above condition implies

$$
\min \left(\frac{j_{t}}{p_{t}}, 1-\frac{j_{t}}{p_{t}}\right) \longrightarrow 0
$$


Assume that $j_{t} / p_{t} \longrightarrow 0$ along some subsequence of $t$. It follows from the definition of the $p_{t}$-adic adding machine that

$$
T^{j_{t}} \rightarrow S
$$

Now we will prove that there exists $a \in G$ such that

$$
\varphi^{\left(j_{t}\right)} \longrightarrow f+a
$$

in measure $\mu$.

The function $\mathrm{f}$ satisfies the condition (see (2) with $\tau=\mathrm{id}$ )

$$
f(T x)-f(x)=\varphi(S x)-\varphi(x) .
$$

The measurability of $f$ and $\xi_{t} \longrightarrow \varepsilon$ imply that there exists $a_{t} \in G$ such that the functions $f_{t}$ defined by

$$
\begin{aligned}
& f_{t}(y)=a_{t}+\varphi^{(i)}(S x)-\varphi^{(i)}(x), \quad y \in D_{i}^{t}, y=T^{i} x, x \in D_{0}^{t}, \\
& \quad i=0, \ldots, p_{t}-1,
\end{aligned}
$$

satisfy the condition

$$
f_{t} \longrightarrow f \text { in measure } \mu \text {. }
$$

We can assume that $a_{t}=b$. We can rewrite (37) as

$$
f_{t}(y)=b+\varphi^{(i)}(S x)-\varphi^{(i)}\left(T^{j_{t}} x\right)+\varphi^{(i)}\left(T^{j_{t}} x\right)-\varphi^{(i)}(x) .
$$

Further we have (see (6))

$$
\varphi^{(i)}\left(T^{j_{t}} x\right)-\varphi^{(i)}(x)=\varphi^{\left(j_{t}\right)}\left(T^{i} x\right)-\varphi^{\left(j_{t}\right)}(x) .
$$

Because of $j_{t}<m_{t}$ then $\varphi^{\left(j_{t}\right)}(x)=b_{t}$ for all $x \in D_{0}^{t}$. Assuming again $b_{t}=b_{1}$ we can write (38) as

$$
\varphi^{(i)}\left(T^{j_{t}} x\right)-\varphi^{(i)}(x)=\varphi^{\left(j_{t}\right)}(y)-b_{1}
$$

and (37) as

$$
f_{t}(y)=b_{2}+\varphi^{\left(j_{t}\right)}(y)+\varphi^{(i)}(S x)-\varphi^{(i)}\left(T^{j_{t}} x\right)
$$

Assume that

$$
x \in D_{u p_{t}}^{t+1}, \quad 0 \leqslant u \leqslant \lambda_{t+1}-1 .
$$

Then

$$
T^{j_{t}} x \in D_{u p_{t}+j_{t}}^{t+1} \quad \text { and } \quad S x \in D_{\left(u+u_{0}\right) p_{t}+j_{t}}^{t+1},
$$

where $u_{0}=q_{0} / r$.

For $i \leqslant p_{t}-j_{t}-1, i=v m_{t}+\rho$ and $u \neq u_{1}, \ldots, u_{r}$ we have

$$
\varphi^{(i)}\left(T^{j_{t}} x\right)=B^{t+1}\left[u p_{t}+j_{t}+i\right]-B^{t+1}\left[u p_{t}+j_{t}\right]=b^{t+1}[u r+v]-b^{t+1}[u r]
$$


and

$$
\begin{aligned}
\varphi^{(i)}(S x) & =B^{t+1}\left[\left(u+u_{0}\right) p_{t}+j_{t}+i\right]-B^{t+1}\left[\left(u+u_{0}\right) p_{t}+j_{t}\right] \\
& =b^{t+1}\left[\left(u+u_{0}\right) r+v\right]-b^{t+1}\left[\left(u+u_{0}\right) r\right] .
\end{aligned}
$$

Thus

$$
\varphi^{(i)}(S x)-\varphi^{(i)}\left(T^{j_{t}} x\right)=\left(b\left[q+q_{0}\right]-b[q]\right)-\left(b\left[u r+q_{0}\right]-b[u r]\right), \quad q=u r+v .
$$

Then (33) and (34) imply

$$
\varphi^{(i)}(S x)-\varphi^{(i)}\left(T^{j_{t}} x\right)=0
$$

except of a set of measure $\leqslant\left(r / \lambda_{t}\right)+\left(j_{t} / p_{t}\right)$.

Now (39) and (40) imply (36) with $a=-b_{2}$. Notice that (35) and (36) and Theorem B imply

$$
T_{\varphi}^{j_{t}} \rightarrow R \circ \sigma_{a}
$$

This proves the lemma.

To prove that

$$
\# \frac{C\left(T_{\varphi}\right)}{\operatorname{wcl}\left\{T_{\varphi}^{n} ; n \in \mathbb{Z}\right\}}=m
$$

in case 3.1 it is sufficient to show that $\sigma_{a} \notin C_{1}$ whenever $a \in \mathbb{Z}_{m}, a \neq 0$. In the case 3.2 we will prove that $\sigma_{a} \in C_{1}$ for every $a \in \mathbb{Z}_{n}$ what implies

$$
\# \frac{C\left(T_{\varphi}\right)}{\operatorname{wcl}\left\{T_{\varphi}^{n} ; n \in \mathbb{Z}\right\}}=1
$$

To do this we need estimations of the $d$-distance between blocks occurring in $\omega$ and $\omega_{t}, t \geqslant 0$.

\section{5. $d$-bar distance between blocks}

The sequence $\omega=b^{0} \stackrel{r}{\times} b^{1} \stackrel{r}{\times} \cdots$ is a concatenation of the blocks of the form

$$
E_{k}(t)=B^{t} \stackrel{r}{\times} \bar{e}_{k}, \quad E_{k}^{(s)}(t)=B^{t} \stackrel{r}{\times} \bar{e}_{k}^{(s)}, \quad k \in \mathbb{Z}_{n}, s=0, \ldots, r-1,
$$

where

$$
\bar{e}_{k}=(\overbrace{k, \ldots, k}^{r}), \quad \bar{e}_{k}^{(s)}=(\overbrace{k, \ldots, k, \underbrace{k+1}_{\text {sth place }}, k, \ldots, k}^{r}) .
$$

The sequence $\omega_{t}=b^{t} \stackrel{r}{\times} b^{t+1} \stackrel{r}{\times} \ldots$ is a concatenation of the blocks of the form

$$
e_{k}(t)=b^{t} \stackrel{r}{\times} \bar{e}_{k}, \quad e_{k}^{(s)}(t)=b^{t} \stackrel{r}{\times} \bar{e}_{k}^{(s)} .
$$


The blocks $E_{k}=E_{k}(t), E_{k}^{(s)}=E_{k}^{(s)}(t)$ are called $t$-symbols and the blocks $e_{k}=$ $e_{k}(t), e_{k}^{(s)}=e_{k}^{(s)}(t)$ are called "small" $t$-symbols. Each fragment $\omega\left[k p_{t},(k+1) p_{t}-1\right]$ of $\omega, k \in \mathbb{Z}$, is a $t$-symbol, and $\omega_{t}\left[k \lambda_{t} r,(k+1) \lambda_{t} r-1\right]$ is a "small" $t$-symbol. The positions $\left[k p_{t},(k+1) p_{t}-1\right]$ and $\left[k \lambda_{t} r,(k+1) \lambda_{t} r-1\right]$ will be called the natural positions in $\omega$ and $\omega_{t}$ respectively.

We will examine $d$-bar distance between the blocks mentioned above or between their special fragments. In particular, we will examine the pairs

$$
b_{k}(i) b_{k}(i+1), b_{k}(i) b_{k+1}(i+1), b_{k+1}(i) b_{k}(i+1),
$$

for $i=0, \ldots, r-2$ and $k \in \mathbb{Z}_{n}$ and

$$
b_{k}(r-1) b_{k}(0), b_{k}(r-1) b_{k+1}(0) .
$$

PROPOSITION 1. - Let

$$
\begin{cases}I=b_{0}^{t}(i)\left[0, \lambda_{t}-j-1\right], & j \leqslant \frac{1}{2} \lambda_{t}, \\ I I=b_{k}^{t}\left(i^{\prime}\right)\left[j, \lambda_{t}-1\right], & k \in \mathbb{Z}_{n}, i, i^{\prime}=0, \ldots, r-1, t \geqslant 0 .\end{cases}
$$

If

$$
d(I, I I)<\frac{1}{r 2^{r+2}}
$$

then $i^{\prime}=i$ and

$$
\begin{gathered}
j=(n-k) r 2^{i+2}+a n r 2^{i+2}, \quad a \geqslant 0, \quad \text { if } 3.1 \text { holds }, \\
j=(n-k) r 4+a n r 4, \quad a \geqslant 0, \quad \text { if } 3.2 \text { holds. }
\end{gathered}
$$

Proof. - It is easy to observe that if $i^{\prime} \neq i$ or $i^{\prime}=i$ and (43) (or (44) in the case 3.2) does not hold then every subblock $F_{k}^{(i)}$ of $I$ differs from the corresponding fragment in $I I$ at least in one position. Since $j \leqslant \frac{1}{2 \lambda_{t}}$, this would imply the converse inequality in (42).

In Propositions $2-6$ the blocks $b_{k}^{t}(i)=b_{k}(i), k \in \mathbb{Z}_{n}, 0 \leqslant i<r$, are those defined in 3.1 .

Proposition 2. - Let

$$
\begin{aligned}
& I=b_{0}(0) \ldots b_{0}(r-1)\left[0, r \lambda_{t}-j-1\right], \\
& I I=b_{k}(0) \ldots b_{k}(r-1)\left[j, r \lambda_{t}-1\right], \quad j \leqslant \frac{1}{2} r \lambda_{t}, \quad k \in \mathbb{Z}_{n} .
\end{aligned}
$$

If

$$
d(I, I I)<\frac{1}{r^{2} 2^{r+3}}
$$

then $j \leqslant \frac{1}{2} \lambda_{t}, k=0$, and

$$
j \equiv 0\left(\bmod n r 2^{r+1}\right) .
$$




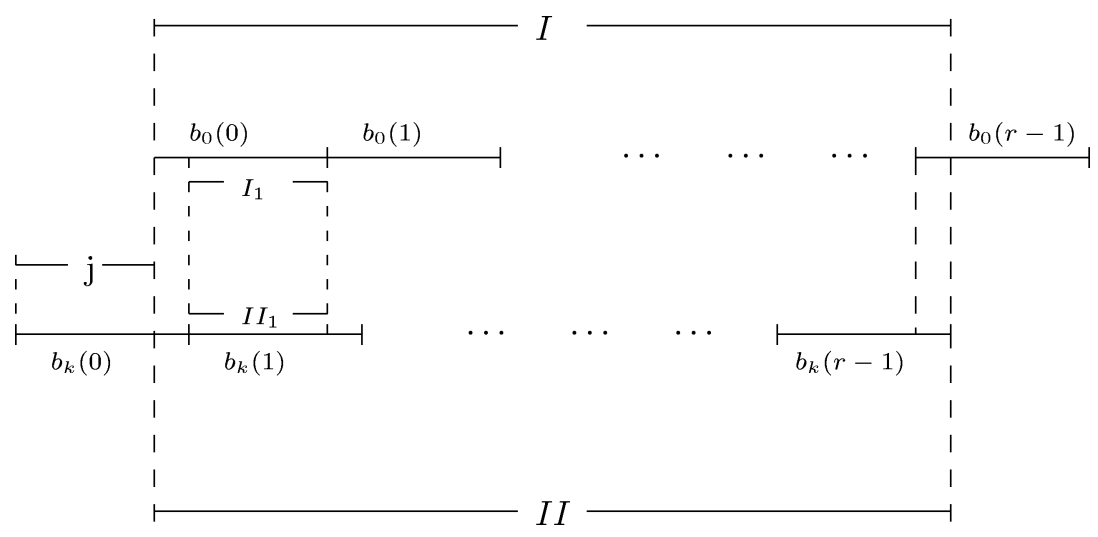

Fig. 1.

Proof. - If $j>\frac{1}{2} \lambda_{t}$ then we can find subblocks $I_{1}$ of $I$ and $I I_{l}$ of $I I$ such that $I I_{1}$ is under $I_{1}$ (see Fig. 1) having the form (41) with different $j^{\prime} s$ and with $i^{\prime} \neq i$.

It follows from Proposition 1 that $d\left(I_{1}, I I_{1}\right) \geqslant \frac{1}{r 2^{r+2}}$ and using (11) we obtain

$$
d(I, I I) \geqslant \frac{\frac{1}{2} \lambda_{t}}{r \lambda_{t}} d\left(I_{1} . I I_{1}\right) \geqslant \frac{1}{r^{2} 2^{r+3}} .
$$

in spite of (45). Therefore $j \leqslant \frac{1}{2} \lambda_{t}$.

It follows from (11) and (45) that

$$
d\left(I_{i}, I I_{i}\right)<\frac{1}{r 2^{r+2}} \quad \text { for } i=0, \ldots, r-1,
$$

where

$$
I_{i}=b_{0}(i)\left[0, \lambda_{t}-j-1\right], \quad I I_{i}=b_{k}(i)\left[j, \lambda_{t}-1\right] .
$$

Then (47) implies (43) to hold for each $i=0, \ldots, r-1$. In particular taking $i=0,1$ we get

$$
-k r 4+2 k r 4=a_{1} n r 4 .
$$

Thus $k=0$ in $\mathbb{Z}_{n}$. The proposition is proved.

Proposition 3. - Let

$$
\begin{gathered}
I=b_{k}(i) b_{k+1}(i+1)[0,2 \lambda-j-1], \quad j \leqslant \frac{1}{2} \lambda ; \lambda=\lambda_{t}, \\
I I=b_{k_{1}}(i) b_{k_{2}}(i+1)[j, 2 \lambda-1], \quad i=0, \ldots, r-2, k, k_{1}, k_{2} \in \mathbb{Z}_{n}
\end{gathered}
$$

and $k_{2}=k_{1}+1$ or $k_{2}=k_{1}-1$.

If

$$
d(I, I I)<\frac{1}{r 2^{r+4}}
$$


then

$$
\left(k_{1} k_{2}\right)=(k, k+1) \quad \text { or } \quad\left(k_{1} k_{2}\right)=(k+4, k+3) \quad \text { if } n \geqslant 3
$$

and

$$
\left(k_{1} k_{2}\right)=(k, k+1) \quad \text { if } n=2 .
$$

Proof. - It follows from (48) and (11) that

$$
d\left(I_{1}, I I_{1}\right)<\frac{1}{r 2^{r+2}}
$$

and

$$
d\left(I_{2}, I I_{2}\right)<\frac{1}{r 2^{r+2}}
$$

where

$$
\begin{gathered}
I_{1}=b_{k}(i)[0, \lambda-j-1], \quad I I_{1}=b_{k_{1}}(i)[j, \lambda-1], \\
I_{2}=b_{k+1}(i+1)[0, \lambda-j-1], \quad I I_{2}=b_{k_{2}}(i+1)[j, \lambda-1] .
\end{gathered}
$$

Now, we apply Proposition 1. It follows from (43) that

$$
k-k_{1}=2\left(k+1-k_{2}\right)(\bmod n) .
$$

The above condition implies (49) and (50).

Proposition 4. - Let

$$
\begin{gathered}
I_{k}=b_{k}(r-1) b_{k}(0)[0,2 \lambda-j-1] \quad \text { or } \quad I_{k}^{\prime}=b_{k}(r-1) b_{k+1}(0)[0,2 \lambda-j-1], \\
I I=b_{k_{1}}(r-1) b_{k_{2}}(0)[j, 2 \lambda-1], \quad k, k_{1}, k_{2} \in \mathbb{Z}_{n}, j \leqslant \frac{1}{2} \lambda_{t},
\end{gathered}
$$

and

$$
k_{2}=k_{1} \quad \text { or } \quad k_{2}=k_{1}+1 \text {. }
$$

If

$$
d(I, I I)<\frac{1}{r 2^{r+4}}, \quad I=I_{k} \text { or } I_{k}^{\prime},
$$

then

$$
k_{1}=k_{2}=k \quad \text { if } I=I_{k} \quad \text { and } \quad k_{1}=k, k_{2}=k+1 \quad \text { if } I=I_{k}^{\prime}
$$

whenever

$$
\left(2^{r-1}-1, n\right)>1,
$$


and there is a unique $l \in \mathbb{Z}_{n}$ such that

$$
\left\{\begin{array}{l}
\left(k_{1} k_{2}\right)=(k k) \quad \text { or } \quad\left(k_{1} k_{2}\right)=(l, l+1) \text { and } l \text { satisfies } \\
l\left(2^{r-1}-1\right)=\left(2^{r-1}-1\right) k+1 \text { in } \mathbb{Z}_{n} \quad \text { if } I=I_{k}, \\
\text { and } \\
\left(k_{1} k_{2}\right)=(k, k+1) \quad \text { or } \quad\left(k_{1} k_{2}\right)=(l l) \text { and } l \text { satisfies } \\
l\left(2^{r-1}-1\right)=\left(2^{r-1}-1\right) k-1 \text { in } \mathbb{Z}_{n} \quad \text { if } I=I_{k}^{\prime},
\end{array}\right.
$$

whenever

$$
\left(2^{r-1}-1, n\right)=1 .
$$

Proof. - Using the same arguments as in the proof of Proposition 3 we obtain from (43)

$$
\left(k_{1}-k\right) 2^{r-1}=k-k_{2}(\bmod n) \quad \text { if } I=I_{k}
$$

and

$$
\left(k_{1}-k\right) 2^{r-1}=k-k_{2}+1(\bmod n) \quad \text { if } I=I_{k}^{\prime} .
$$

The above, (51), (53) and (55) imply (52) and (54) respectively.

The next proposition is an easy consequence of (9) and the definition of the blocks $b(0), \ldots, b(r-1)$.

\section{PROPOSITION 5. - Let}

$$
\begin{gathered}
I_{l}=b_{l}(i)\left[0, \lambda_{t}-j-1\right], \quad I I_{k}=b_{k}(i)\left[j, \lambda_{t}-1\right], \\
j \leqslant \frac{1}{2} \lambda_{t}, k, l \in \mathbb{Z}_{n}, 0 \leqslant i \leqslant r-1 .
\end{gathered}
$$

If $j \equiv 0\left(\bmod n r 2^{r+1}\right)$ and $k \neq l$ then

$$
d\left(I_{l}, I I_{k}\right)=1
$$

Proposition 6. - Let

$$
I=b^{t} \stackrel{r}{\times} C, \quad I I=b^{t} \stackrel{r}{\times} D\left[j, j+\lambda_{t}|D|-1\right], \quad 0 \leqslant j \leqslant r \lambda_{t}-1,
$$

where $|C| \geqslant 3 r,|D|=|C|+r, C, D \subset \omega_{t+1}\left(\right.$ see (21)) and $C=\omega_{t+1}[p r, p r+|C|-$ $1], D=\omega_{t+1}[q r, q r+|D|-1]$. If

$$
d(I, I I)<\delta, \quad \delta<\frac{1}{3 r^{2} 2^{r+3}},
$$

then either

$$
j<\delta r 2^{r+1} \lambda_{t} \quad \text { and } \quad d\left(C, D_{1}\right)<\delta
$$

or

$$
r \lambda_{t}-\delta r 2^{r+1} \lambda_{t}<j \leqslant r \lambda_{t} \text { and } d\left(C, D_{1}\right)<\delta
$$


where

$$
\begin{aligned}
& D_{1}=D[0,|D|-r-1] \quad \text { if } j \leqslant \frac{1}{2} r \lambda_{t}, \\
& D_{1}=D[r,|D|-1] \quad \text { if } j>\frac{1}{2} r \lambda_{t} .
\end{aligned}
$$

Proof. - We can represent $C$ and $D$ as

$$
C=C_{1} C_{2} \ldots C_{s}, \quad D=D_{1} D_{2} \ldots D_{s} D_{s+1},
$$

where

$$
\left|C_{1}\right|=\cdots=\left|C_{s}\right|=\left|D_{1}\right|=\cdots=\left|D_{s}\right|=\left|D_{s+1}\right|=r, s \geqslant 3,
$$

and every $C_{1}, \ldots, C_{s}, D_{1}, \ldots, D_{s+1}$ is equal to one of the blocks $\bar{e}_{k}, \bar{e}_{k}^{(v)}, k \in \mathbb{Z}_{n}, v=$ $0, \ldots, r-1$ (see 3.5). Assume that $j \leqslant \frac{1}{2} r \lambda_{t}$. Using (12) we get

$$
d(I, I I)=\frac{1}{s} \sum_{p=1}^{s}\left(b \stackrel{r}{\times} C_{p}, A_{p}\right),
$$

where

$$
A_{p}=\left(b \stackrel{r}{\times} D_{p}\right)\left(b \stackrel{r}{\times} D_{p+1}\right)\left[j, j+r \lambda_{t}-1\right] .
$$

Then (56) implies that

$$
d\left(b \stackrel{r}{\times} C_{p}, A_{p}\right)<\frac{1}{3 r^{2} 2^{r+3}}
$$

for at least one $p$. Using the same arguments as in the proof of Proposition 2 we obtain $j \leqslant \frac{1}{2} \lambda_{t}$.

Let

$$
Q=\left\{1 \leqslant p \leqslant s, C_{p} \text { and } D_{p} \text { are equal } \bar{e}_{k}, \bar{e}_{l} \text { for some } k, l \in \mathbb{Z}_{n}\right\} .
$$

It follows from the definitions of $\omega, \omega_{t}$ and $b^{t}$ s that

$$
\# Q \geqslant \frac{1}{3} s .
$$

This inequality, (56), and (59), imply

$$
\frac{1}{|Q|} \sum_{p \in Q} d\left(b \stackrel{r}{\times} C_{p}, A_{p}\right)<\frac{1}{r^{2} 2^{r+3}} .
$$

Now we conclude that there is at least one $p \in Q$ such that

$$
d\left(b \stackrel{r}{\times} C_{p}, A_{p}\right)<\frac{1}{r^{2} 2^{r+3}} .
$$

It follows from Proposition 2 that $j \equiv 0\left(\bmod n r 2^{r+1}\right)$. 


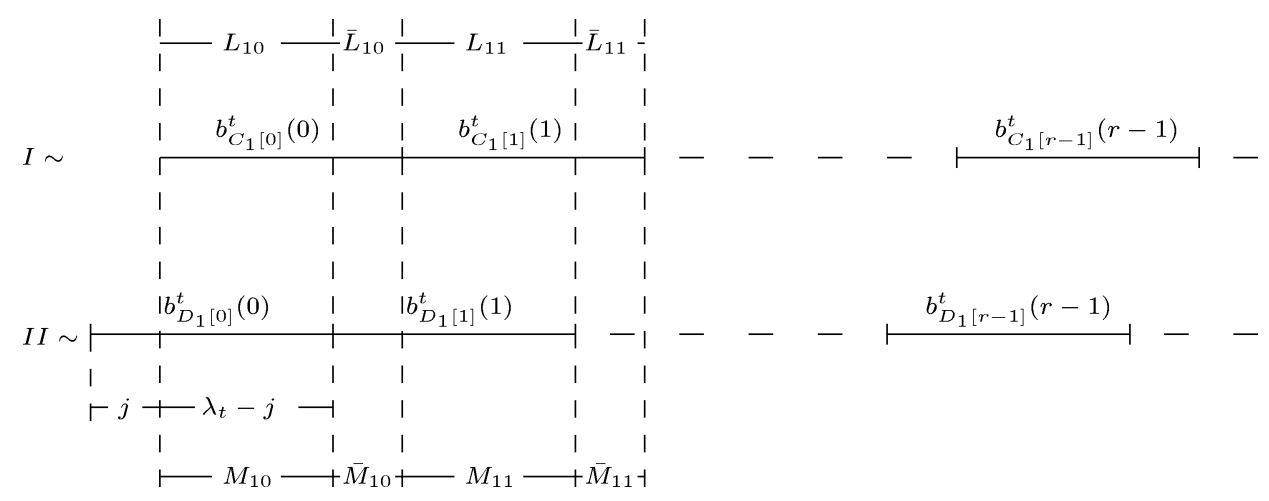

Fig. 2.

Now, using (10) and (12) again we get (see Fig. 2)

$$
d(I, I I)=\frac{1}{r} \sum_{i=0}^{r-1} \frac{1}{s}\left(\left(1-\frac{j}{\lambda_{t}}\right) \sum_{u=1}^{s} d\left(L_{u i}, M_{u i}\right)+\frac{j}{\lambda_{t}} \sum_{u=1}^{s} d\left(\bar{L}_{u i}, \bar{M}_{u i}\right)\right),
$$

where

$$
\begin{array}{ll}
L_{u i}=b_{C_{u}[i]}^{t}(i)\left[0, \lambda_{t}-j-1\right], & M_{u i}=b_{D_{u}[i]}^{t}(i)\left[j, \lambda_{t}-1\right], \\
\bar{L}_{u i}=b_{C_{u}[i]}^{t}(i)\left[\lambda_{t}-j, \lambda_{t}-1\right], & \bar{M}_{u i}=b_{D_{u}[i]}^{t}(i+1)[0, j-1] .
\end{array}
$$

It is not hard to remark that if $j \neq 0$

$$
d\left(\bar{L}_{u i}, \bar{M}_{u i}\right) \geqslant \frac{1}{r 2^{r+1}}
$$

for every $u$ and $i, 1 \leqslant u \leqslant s, 0 \leqslant i \leqslant r-1$. Let

$$
a=\#\{0 \leqslant k \leqslant|C|-1, C[k] \neq D[k]\} .
$$

Then using Proposition 5, (60) and (61) we get

$$
\delta>d(I, I I) \geqslant \frac{a}{|C|}\left(1-\frac{j}{\lambda_{t}}+\frac{j}{\lambda_{t}} \frac{1}{r 2^{r+1}}\right) .
$$

The above gives

$$
\delta>\frac{a}{|C|}\left(1-\frac{j}{\lambda_{t}}\right) \geqslant \frac{a}{|C|} \frac{1}{2}
$$

and then $\frac{a}{|C|}<2 \delta$. This inequality, (56) and (62) imply

$$
\delta>\frac{a}{|C|}+\frac{j}{\lambda_{t}}\left(\frac{1}{r 2^{r+1}}-\frac{a}{|C|}\right)>\frac{a}{|C|}+\frac{j}{\lambda_{t}}\left(\frac{1}{r 2^{r+1}}-2 \delta\right)>\frac{a}{|C|}=d\left(C, D_{1}\right) .
$$


We have obtained the second inequality of (57). To get the first inequality of (57) we use (62) to obtain

$$
\delta>\frac{j}{\lambda_{t}} \frac{1}{r 2^{r+1}} .
$$

This implies (57). We have proved the proposition if $j \leqslant \frac{1}{2} r \lambda_{t}$. The case $\frac{1}{2} r \lambda_{t}<j<r \lambda_{t}$ leads to (58) in a similar way. The proposition is proved.

PROPOSITION 7. - Let

$$
I=B^{t} \stackrel{r}{\times} C, \quad I I=B^{t} \stackrel{r}{\times} D\left[j, j+m_{t}|D|-1\right], \quad 0 \leqslant j \leqslant p_{t}-1,
$$

where $C$ and D satisfy the same conditions as in Proposition 6. If

$$
d(I, I I)<\delta, \quad \delta<\frac{1}{3 r^{2} 2^{r+3}},
$$

then either

$$
j<\delta r 2^{r+} p_{t} \quad \text { and } \quad d\left(C, D_{1}\right)<\delta
$$

or

$$
p_{t}-\delta r 2^{r+1} p_{t}<j<p_{t} \quad \text { and } \quad d\left(C, D_{1}\right)<\delta,
$$

where

$$
D_{1}=D[0,|D|-r-1] \quad \text { if } j \leqslant \frac{1}{2} p_{t},
$$

and

$$
D_{1}=D[r,|D|-1] \quad \text { if } r>\frac{1}{2} p_{t} .
$$

Proof. - We use an induction argument and can repeat the proof of Lemma 3 from [8, p. 198], using (8), (9), and also using Proposition 6 instead of using a Lemma 2 as in [8, p. 196].

\section{6. $d$-bar distance between blocks - the case 3.2}

Using the same methods as in 3.5 we can estimate the distance between blocks $b_{k}^{t}(i)$ and $B_{k}^{t}(i), i=0, \ldots, r-1, k \in \mathbb{Z}_{n}, t \geqslant 0$, defined in the case 3.2.

As an easy consequence of Proposition 1 we get

PROPOSITION 8. - Let

$$
\begin{aligned}
I_{l} & =b_{l_{0}}(0) \ldots b_{l_{r-1}}(r-1)\left[0, r \lambda_{t}-j-1\right], \\
I I_{k} & =b_{k_{0}}(0) \ldots b_{k_{r-1}}(r-1)\left[j, r \lambda_{t}-1\right],
\end{aligned}
$$

$j \leqslant \frac{1}{2} r \lambda_{t}$, where $\left(l_{0}, \ldots, l_{r-1}\right)$ (resp. $\left.\left(k_{0}, \ldots, k_{r-1}\right)\right)$ is of the form $\bar{e}_{l}$ or $\bar{e}_{l}^{(v)}$ (resp. $\bar{e}_{k}$ or $\left.\bar{e}_{k}^{\left(v^{\prime}\right)}\right), k, l \in \mathbb{Z}_{n}$ and $v, v^{\prime}=0, \ldots, r-1$. If

$$
d\left(I_{l}, I I_{k}\right)<\frac{1}{r^{2} 2^{r+3}}
$$


then $j \leqslant \frac{1}{2} \lambda_{t}$ and there is a unique $s \in \mathbb{Z}_{n}, s=s(t)$, such that $l_{i}=k_{i}+s$ for every $i=0, \ldots, r-1$. Moreover $j$ has a form

$$
j=(n-s) r 4+a n r 4, \quad a \geqslant 0 .
$$

As an analogue of Proposition 5 we obtain

Proposition 9. - Let $I_{l}, I I_{k}$ be as in Proposition 5,

$$
j \leqslant \frac{1}{2} \lambda_{t} \quad \text { and } \quad j \equiv(n-s)(\bmod 4 r n)
$$

for some $s \in \mathbb{Z}_{n}$. Then

$$
d\left(I_{l}, I I_{k}\right)=1 \quad \text { whenever } k-l \neq s .
$$

Then using Propositions 8 and 9 we have

Proposition 10. - Let I and II be as in Proposition 6 and

$$
\begin{aligned}
& |C| \geqslant r, \quad|D|=|C|+r, \quad C, D \subset \omega_{t+1}, \\
& C=\omega_{t+1}[p r, p r+|C|-1], \quad D=\omega_{t+1}[q r, q r+|D|-1] .
\end{aligned}
$$

If

$$
d(I, I I)<\delta, \quad \delta<\frac{1}{3 r^{2} 2^{r+3}},
$$

then there is an unique $s \in \mathbb{Z}_{n}, s=s(t)$, such that

$$
j<\delta r 2^{r+1} \lambda_{t} \text { and } d\left(C, D_{1}\right)<\delta
$$

or

$$
r \lambda_{t}-\delta r 2^{r+1} \lambda_{t}<j \leqslant r \lambda_{t} \text { and } d\left(C, D_{1}\right)<\delta,
$$

where $D_{1}=D[0,|D|-r-1]=C+s$ if $j \leqslant \frac{1}{2} \lambda_{t} r$, and $D_{1}=D[r,|D|-1]=C+s$ if $j>\frac{1}{2} r \lambda_{t}$.

Using arguments as in Lemma 3 in [8] and Proposition 10 we get

Proposition 11. - Let I and II be as in the Proposition 7 and C, D satisfy the same conditions as in Proposition 10.

If

$$
d(I, I I)<\delta, \quad \delta<\frac{1}{3 r^{2} 2^{r+3}},
$$

there exists an unique $s \in \mathbb{Z}_{n}, s=s(t)$, such that either

$$
j<\delta r 2^{r+1} p_{t} \text { and } d\left(C, D_{1}\right)<\delta
$$

or

$$
p_{t} \delta r 2^{r+1} p_{t}<j<p_{t} \quad \text { and } \quad d\left(C, D_{1}\right)<\delta,
$$


where

and

$$
D_{1}=D[0,|D|-r-1]+s \quad \text { if } j \leqslant \frac{1}{2} p_{t}
$$

$$
D_{1}=D[r,|D|-1]+s \quad \text { if } j>\frac{1}{2} p_{t} .
$$

\subsection{The centralizer of $T_{\varphi}$ (continuation)}

In 3.4 we have proved that $C\left(T_{\varphi}\right)$ consists of the elements $R \circ \sigma_{a}$, where $R$ is a limit of powers of $T_{\varphi}$ and $\sigma_{a}$ is defined by (4), $a \in \mathbb{Z}_{n}$. Now we are in a position to show that

$$
\# \frac{C\left(T_{\varphi}\right)}{\operatorname{wcl}\left\{T_{\varphi}^{n} ; n \in \mathbb{Z}\right\}}= \begin{cases}n & \text { in the case } 3.1 \\ 1 & \text { in the case 3.2. }\end{cases}
$$

LEMMA 3. - If the case 3.1 holds and $\sigma_{a} \in C_{1}$ then $a=0$.

Proof. - Let us suppose that $T_{\varphi}^{n_{s}} \rightarrow \sigma_{a}, a \in \mathbb{Z}_{n}$. Then Corollary 1 says that $\varphi^{\left(n_{s}\right)} \longrightarrow a$ in measure. Let

$$
\varepsilon_{s}=\mu\left\{x \in X ; \varphi^{\left(n_{s}\right)}(x) \neq a\right\} .
$$

We have $\varepsilon_{s} \longrightarrow 0$. Now for every $s$ find $t_{s}$ such that

$$
\frac{n_{s}}{p_{t_{s}}}<\frac{\varepsilon_{s}}{r}
$$

To shorten notation we let $t:=t_{s}+1, \bar{t}:=t_{s}$. Take $x \in D_{j}^{t}$. Then using (18) we get

$$
\varphi^{\left(n_{s}\right)}(x)=B^{t}\left[j+n_{s}\right]-B^{t}[j]
$$

except of $j$ 's satisfying $m_{t}-1-n_{s} \leqslant j \leqslant m_{t}-1,2 m_{t}-1-n_{s} \leqslant j \leqslant 2 m_{t}-$ $1, \ldots, p_{t}-1-n_{s} \leqslant j \leqslant p_{t}-1$. Then (63) and (64) imply

$$
\frac{1}{p_{t}} \#\left\{0 \leqslant j \leqslant p_{t}-1, B^{t}\left[j+n_{s}\right]-B^{t}[j] \neq a\right\}<\varepsilon_{s}+\varepsilon_{s}=2 \varepsilon_{s} .
$$

This means that

$$
d\left(B^{t}\left[0, p_{t}-n_{s}-1\right], B_{-a}^{t}\left[n_{s}, p_{t}-1\right]\right)<2 \varepsilon_{s}
$$

We can write

$$
B^{t}=B^{\bar{t}} \stackrel{r}{\times} b^{t}, \quad B_{-a}^{t}=B^{\bar{t}} \stackrel{r}{\times} b_{-a}^{t} .
$$

If $\varepsilon_{s}<\frac{1}{6 r^{2} 2^{r+3}}$ then we apply Proposition 7 to the blocks $I=B^{\bar{t}} \stackrel{r}{\times} b^{t}$ and $I I=B^{\bar{t}} \stackrel{r}{\times} b_{-a}^{t}$. As a consequence we obtain

$$
d\left(b^{t}, b_{-a}^{t}\right)<2 \varepsilon_{s} .
$$

This equality implies (Proposition 2) $a=0$. The lemma is proved.

From Lemmas 2 and 3 we obtain 
THEOREM 2.-

$$
\# \frac{C\left(T_{\varphi}\right)}{\operatorname{wcl}\left\{T_{\varphi}^{n}, n \in \mathbb{Z}\right\}}=n
$$

if the case 3.1 holds.

Now, we examine the case 3.2. It follows from the definition of the blocks $b_{0}(i)=$ $b_{0}^{t}(i), i=0, \ldots, r-1, a \in \mathbb{Z}_{n}$ that

$$
b(i)[(n-a) 4 r, \lambda-1]=b_{a}(i)[0, \lambda-(n-a) 4 r-1],
$$

for every $i=0, \ldots, r-1$.

Set $n_{t}=(n-a) 4 r p_{t-1}$. Then (66) implies

$$
B^{t}(i)\left[j+n_{t}\right]-B^{t}(i)[j]=a
$$

for $j=0, \ldots, p_{t}-n_{t}-1$, and $i=0, \ldots, r-1$. (65) and the above imply $\varphi^{\left(n_{t}\right)}(x)=a$ except of a set of measure $<r \frac{n_{t}}{p_{t}} \leqslant \frac{4 r^{2} n}{\lambda_{t}}$.

Hence $\varphi^{\left(n_{t}\right)} \longrightarrow a$ in measure which implies that $T_{\varphi}^{n_{t}} \rightarrow \sigma_{a}, a \in \mathbb{Z}_{n}$. We have shown that $\sigma_{a} \in C_{1}$ for every $a \in \mathbb{Z}_{n}$ and as a consequence of Lemma 2 we get

THEOREM 3.-

$$
\# \frac{C\left(T_{\varphi}\right)}{\operatorname{wcl}\left\{T_{\varphi}^{n} ; n \in \mathbb{Z}\right\}}=1
$$

if the case 3.2 holds.

Theorem $3^{\prime} .-\operatorname{wcl}\left\{T_{\varphi}^{n}, n \in Z\right\}$ is uncountable.

Proof. - Let

$$
g_{0}=\sum_{0}^{\infty} u_{t} p_{t-1}, \quad u_{t}=w_{t}\left(r m 2^{r+1}\right)
$$

in the case (3.1) and $u_{t}=w_{t}(4 r n)$ in the case (3.2) $0 \leqslant u_{t} \leqslant r \lambda_{t}-1$ and assume that

$$
\sum_{t=0}^{\infty} \min \left(\frac{w_{t}}{r 2^{t}}, 1-\frac{w_{t}}{r 2^{t}}\right)<\infty
$$

Repeating the same arguments as in Lemma 4 of [9] we can construct a measurable function $f: X \longrightarrow G$ such that

$$
f(T x)-f(x)=\varphi(S x)-\varphi(x), \quad \text { for a.e. } x \in X .
$$

Thus the triple $R=(S, f$, id $) \in C\left(T_{\varphi}\right)$. Of course, there is a continuum of $g_{0}$ 's in $X$ satisfying the above conditions. Hence $C\left(T_{\varphi}\right)$ is uncountable. Then Theorem 2 and 3 imply $\operatorname{wcl}\left\{T_{\varphi}^{n}, n \in Z\right\}$ is uncountable. 


\section{Rank of $T_{\varphi}$ is $r$}

In this section we use the shift representation $\left(\Omega_{\omega}, T_{\sigma}\right)$ of $\left(X \times \mathbb{Z}_{n}, T \varphi\right)$ (see 2.3) and the definition of rank given at the end of 2.2.

We will also require the notion of $\delta$-cover: let $\mathcal{A}$ be a (finite) family of blocks and $B$ a block such that $|B| \in\{|A|: A \in \mathcal{A}\}$, we let

$$
d(B, \mathcal{A})=\min \{d(B, A): A \in \mathcal{A},|A|=|B|\} .
$$

If $\mathcal{A}=\left\{A_{1}, \ldots, A_{k}\right\}, C$ is a block, and $\delta>0$, we define

$$
t_{\delta}(\mathcal{A}, C)=t_{\delta}\left(A_{1}, \ldots, A_{k}, C\right)=\max \left\{\frac{\left|C_{1}\right|+\cdots+\left|C_{p}\right|}{|C|}\right\},
$$

where the maximum is taken over all concatenations of the form

$$
C=\epsilon_{1} C_{1} \epsilon_{2} \ldots \epsilon_{p} C_{p} \epsilon_{p+1}
$$

for which $d\left(C_{i}, \mathcal{A}\right)<\delta, 1 \leqslant i \leqslant p$. Then we define, for a strictly ergodic one-sided sequence $\omega$,

$$
t_{\delta}(\mathcal{A}, \omega)=\liminf _{N \rightarrow \infty} t_{\delta}(\mathcal{A}, \omega[0, N])\left(=\lim _{N \rightarrow \infty} t_{\delta}(\mathcal{A}, \omega[0, N])\right) .
$$

In particular, $t_{\delta}(A, \omega)$ is defined for a single block $A$, or if $\omega=C$ is finite.

It is known $([3,20])$ that in the case under consideration the rank of $\left(\Omega_{\omega}, S, \mu_{\omega}\right)$ is at most $r$ if for any $\delta>0$ and any $N \in \mathbb{N}$, there exists $\mathcal{A}$ of cardinality $r$ such that $|A| \geqslant N$, $A \in \mathcal{A}$, and

$$
t_{\delta}(\mathcal{A}, \omega) \geqslant 1-\delta .
$$

This definition agrees with that of sub-section 2.2.

Given a one-sided $\eta$, some $\delta>0$, and a family $\mathcal{A}$ of blocks, we will say that the subsequence $\tilde{\eta}$ of $\eta$ (finite or infinite) is $\delta$-covered by $\mathcal{A}$ if $t_{\delta}(\tilde{\eta}, \mathcal{A}) \geqslant 1-\delta$.

\subsection{The frequencies of $t$-symbols and an estimation of the rank}

Let $\operatorname{Fr}(E, \omega)$ be the average frequency of a $t$-symbol $E$ (see 3.5) appearing in $\omega$ at natural positions. Similarly, let $\operatorname{Fr}\left(e, \omega_{t}\right)$ denote the average frequency of a "small" $t$ symbol $e$ appearing in $\omega_{t}$ at natural positions. It is easy to get the following equalities;

$$
\left\{\begin{array}{l}
\operatorname{Fr}\left(E_{k}, \omega\right)=\operatorname{Fr}\left(e_{k}, \omega_{t}\right)=\frac{1}{r n} \sum_{i=0}^{r-1}\left(1-\frac{1}{2^{i+2}}\right)=\frac{1}{n}\left[1-\frac{1}{r} \sum_{i=0}^{r-1} \frac{1}{2^{i+2}}\right] \\
\text { and } \\
\operatorname{Fr}\left(E_{k}^{(s)}, \omega\right)=\operatorname{Fr}\left(e_{k}^{(s)}, \omega_{t}\right)=\frac{1}{r n 2^{s+2}}, \quad s=0, \ldots, r-1, k \in \mathbb{Z}_{n},
\end{array}\right.
$$


if the case 3.1 holds. In the case 3.2 we have

$$
\left\{\begin{array}{l}
\operatorname{Fr}\left(E_{k}, \omega\right)=\operatorname{Fr}\left(e_{k}, \omega_{t}\right)=\frac{3}{4 n}, \\
\operatorname{Fr}\left(E_{k}^{(s)}, \omega\right)=\operatorname{Fr}\left(e_{k}^{(s)}, \omega_{t}\right)=\frac{1}{4 n r}, \quad k \in \mathbb{Z}_{n}, s=0, \ldots, r-1 .
\end{array}\right.
$$

PROPOSITION 12. $-r\left(T_{\varphi}\right) \leqslant r$.

Proof. - Consider the blocks

$$
L_{k}^{(s)}=L_{k}^{(s)}(t)=B^{t} \stackrel{r}{\times} b_{k}^{t+1}(s), \quad s=0, \ldots, r-1, t \geqslant 0, k \in \mathbb{Z}_{n} .
$$

We have

$$
E_{k}=L_{k}^{(0)} \ldots L_{k}^{(r-1)}, \quad E_{k}^{(s)}=L_{k}^{(0)} \ldots L_{k}^{(s-1)} L_{k+1}^{(s)} L_{k}^{(s+1)} \ldots L_{k}^{(r-1)}
$$

for every $k \in \mathbb{Z}_{n}$ and $s=0, \ldots, r-1$.

Because the blocks $E_{k}, E_{k}^{(s)}$ cover completely the sequence $\omega$ then the blocks $L_{k}^{(0)} \ldots L_{k}^{(r-1)}, k \in \mathbb{Z}_{n}$, also cover $\omega$.

We know that

$$
\begin{aligned}
& b^{t+1}(s)\left[0, \lambda_{t+1}-k n r 2^{r+1}\right]=b_{-k}^{t+1}(0)\left[k n r 2^{r+1}, \lambda_{t+1}-1\right], \\
& k \in \mathbb{Z}_{n}, \quad s=0, \ldots, r-1, \quad \text { if } 3.1 \text { holds },
\end{aligned}
$$

and

$$
\begin{aligned}
& b^{t+1}(s)\left[0, \lambda_{t+1}-k n r 4\right]=b_{-k}^{t+1}(0)\left[k n r 4, \lambda_{t+1}-1\right], \\
& k \in \mathbb{Z}_{n}, \quad s=0, \ldots, r-1, \quad \text { if } 3.2 \text { holds. }
\end{aligned}
$$

The last equalities imply that the block $L_{0}^{(s)}$ cover each block $L_{k}^{(s)}, k \in \mathbb{Z}_{n}$, except of a part with the length $\leqslant n^{2} 2^{r+1} p_{t}$ in the case 3.1 and $\leqslant n^{2} 4 p_{t}$ in the case 3.2, for $s=0, \ldots, r-1$. Thus the blocks $L_{0}^{(0)}, \ldots, L_{0}^{(r-1)}$ cover the sequence $\omega$ except of a part with the density $\leqslant n^{2} 2^{r+1} / \lambda_{t+1}$ if 3.1 holds and $\leqslant n^{2} 4 / \lambda_{t+1}$ if 3.2 holds. Simultaneously $\left|L_{0}^{(s)}(t)\right| \stackrel{t \rightarrow \infty}{\longrightarrow} \infty$. According to the definition of the rank (see 2.2) we have $r\left(T_{\varphi}\right) \leqslant r$.

\subsection{Special subblocks of $\omega_{t}$}

Fix $t \geqslant 0$. We distinguish special subblocks $C$ of $\omega_{t}$ of the form $b^{t} \stackrel{r}{\times} \bar{C}$, where $\bar{C}$ is a strict subblock of one of the following blocks (cf. 3.5)

$$
\left\{\begin{array}{l}
e_{k} e_{k}, e_{k} e_{k}^{(s)}, e_{k}^{(s)} e_{k+1}, \quad k \in \mathbb{Z}_{n}, s=0, \ldots, r-1, \\
\text { where } e_{k}=e_{k}(t+1), e_{k}^{(s)}=e_{k}^{(s)}(t+1), \\
\text { if the case } 3.2 \text { is considered, }
\end{array}\right.
$$

or

$$
\left\{\begin{array}{l}
e_{k} e_{k} e_{k} e_{k}, e_{k} e_{k} e_{k} e_{k}^{(s)}, e_{k} e_{k} e_{k}^{(s)} e_{k+1}, e_{k} e_{k}^{(s)} e_{k+1} e_{k+1}, e_{k}^{(s)} e_{k+1} e_{k+1} e_{k+1}, \\
k \in \mathbb{Z}_{n}, s=0, \ldots, r-1 \\
\text { if the case } 3.1 \text { is considered. }
\end{array}\right.
$$


Notice that blocks (69) are all pairs of "small" $(t+1)$-symbols appearing in $\omega_{t+1}$, as well as the blocks (70) are all possible quadruples of "small" $(t+1)$-symbols appearing in $\omega_{t+1}$. Let us list the different cases we shall deal with afterwards:

(A) $\bar{C} \subset b_{k_{0}}^{t+1}\left(i_{0}\right)$ for some $k_{0} \in \mathbb{Z}_{n}$ and $i_{0}=0, \ldots, r-1$ (cases 3.1 or 3.2);

(B) (the case 3.2) $\bar{C}=b_{k_{i_{0}}}\left(i_{0}\right) \ldots b_{k_{r-1}}(r-1) \mid b_{l_{0}} \ldots b_{l_{i_{1}}}\left(i_{1}\right)$ where $b(i)=b^{t+1}(i)$, $i_{0}>0, i_{1}<r-1$.

$E:=\left(k_{i_{0}} \ldots k_{r-1} l_{0} \ldots l_{i_{1}}\right)$ is contained in one of the following blocks;

$$
\bar{e}_{k} \bar{e}_{k}, \bar{e}_{k} \bar{e}_{k}^{(s)}, \bar{e}_{k}^{(s)} \bar{e}_{k+1}, \quad k \in \mathbb{Z}_{n}, s=0, \ldots, r-1,
$$

and $2 \leqslant|E|<2 r$

$\left(\mathrm{B}^{\prime}\right)$ (the case 3.1) $\bar{C}=b_{k_{i_{0}}}\left(i_{0}\right) . . b_{k_{r-1}}(r-1)\left|b_{u_{0}}(0) . . b_{u_{r-1}}(r-1)\right| b_{v_{0}}(0) . . b_{v_{r-1}}(r-$ 1) $\mid b_{l_{0}}(0) . . b_{l_{i_{1}}}\left(i_{1}\right)$ and $E=\left(k_{i_{0}} \ldots k_{r-1}\left|u_{0} \ldots u_{r-1}\right| v_{0} \ldots v_{r-1} \mid l_{0} \ldots l_{i_{1}}\right), 2 \leqslant|E|<$ $4 r, i_{0}>0, i_{1}<r-1$, is contained in one of the blocks

$$
\bar{e}_{k} \bar{e}_{k} \bar{e}_{k} \bar{e}_{k}, \bar{e}_{k} \bar{e}_{k} \bar{e}_{k} \bar{e}_{k}^{(s)}, \bar{e}_{k} \bar{e}_{k} \bar{e}_{k}^{(s)} \bar{e}_{k+1}, \bar{e}_{k} \bar{e}_{k}^{(s)} \bar{e}_{k+1} \bar{e}_{k+1}, \bar{e}_{k}^{(s)} \bar{e}_{k+1} \bar{e}_{k+1} \bar{e}_{k+1} \text {. }
$$

In general we can write

$$
\bar{C}=\bar{C}_{1} \bar{C}_{2} \bar{C}_{3}
$$

where $\bar{C}_{2}$ is as in (A) or as in (B) (the case 3.2) or ( $\left.\mathrm{B}^{\prime}\right)$ (the case 3.1), and

$$
\left\{\begin{array}{l}
\bar{C}_{1}=b_{k^{\prime}}^{t+1}\left(i_{0}-1\right)\left[l_{1} r, \lambda-1\right], \quad \bar{C}_{3}=b_{k^{\prime \prime}}^{t+1}\left(i_{1}+1\right)\left[0, l_{2} r-1\right], \\
0<l_{1} \leqslant \lambda-1,0<l_{2} \leqslant \lambda-1, \lambda=\lambda_{t+1},
\end{array}\right.
$$

and $k^{\prime} E k^{\prime \prime}$ is contained in one of the blocks (71) or (72) respectively ( $E$ is defined by $\bar{C}_{2}$ ).

Then we can distinguish the next special kinds of blocks (73) for given $\delta>0$ :

(G1) $\left|\bar{C}_{1}\right| /|\bar{C}|>\delta$ and $\left|\bar{C}_{3}\right| /|\bar{C}|>\delta$,

(G2) $\left|\bar{C}_{1}\right| /|\bar{C}|>\delta$ and $\left|\bar{C}_{3}\right| /|\bar{C}| \leqslant \delta$,

(G3) $\left|\bar{C}_{1}\right| /|\bar{C}| \leqslant \delta$ and $\left|\bar{C}_{3}\right| /|\bar{C}|>\delta$,

(G4) $\left|\bar{C}_{1}\right| /|\bar{C}| \leqslant \delta$ and $\left|\bar{C}_{3}\right| /|\bar{C}| \leqslant \delta$.

\section{3. $r\left(T_{\varphi}\right)=r$ : the case 3.2}

Take $0<\delta^{2}<1 /\left(r^{2} 2^{2 r+3}\right)$.

Proposition 13. - Assume that $\bar{C}$ is as in (B) and let $d(C, D)<\delta^{2}, D \subset \omega_{t}$. Then $D$ has a form

$$
D=\left(b^{t} \stackrel{r}{\times} \bar{D}\right)[j, j+|D|-1], \quad \text { where } \bar{D} \subset \omega_{t+1}
$$

and

$$
\left\{\begin{array}{c}
\bar{D}=b_{k_{i_{0}}^{\prime}}^{t+1}\left(i_{0}\right) \ldots b_{k_{r-1}^{\prime}}^{t+1}(r-1) \mid b_{l_{0}^{\prime}}^{t+1}(0) \ldots b_{l_{i_{1}}^{\prime}}^{t+1}\left(i_{1}\right) b_{l_{i_{1}+1}^{\prime}}^{t+1}\left(i_{1}+1\right), \\
\text { and } j<\delta^{2} r 2^{r+1} \lambda_{t+1}, l_{i_{1}+1}^{\prime} \in \mathbb{Z}_{n}
\end{array}\right.
$$


or

$$
\left\{\begin{array}{l}
\bar{D} \text { is as in (76) and } \\
j>r \lambda_{t+1}-\delta^{2} r 2^{r+1} \lambda_{t+1}
\end{array}\right.
$$

Moreover, there is a unique $s_{0} \in \mathbb{Z}_{n}$ such that

$$
\left(k_{0}^{\prime} \ldots k_{r-1}^{\prime} \mid l_{0}^{\prime} \ldots l_{i_{1}}^{\prime}\right)=\left(k_{0} \ldots k_{r-1} \mid l_{0} \ldots l_{i_{1}}\right)+s_{0}
$$

if (76) holds and

$$
\left(k_{1}^{\prime} \ldots k_{r-1}^{\prime} \mid l_{0}^{\prime} \ldots l_{i_{1}+1}^{\prime}\right)=\left(k_{0} \ldots k_{r-1} \mid l_{0} \ldots l_{i_{1}}\right)+s_{0}
$$

if (77) holds.

Proof. - The proposition is an easy consequence of the Proposition 10 where $t$ is taken instead of $t+1\left(\delta^{2}<1 /\left(r^{2} 2^{2 r+3}\right)<1 /\left(3 r^{2} 2^{r+3}\right)\right)$.

Given a block $A \subset \omega$ or $\omega_{t}, A=\omega[l, l+|A|-1]$ we define $A(\delta)$ as $A(\delta)=$ $\omega[l-\delta|A|, l+|A|+\delta|A|-1], \delta>0$. The next proposition says that if $C$ is as in (G1), (G2), (G3), or (G4), there is a block $C^{\prime}=b^{t} \stackrel{r}{\times} \widetilde{C}$ such that $\widetilde{C}$ is as in (B) and either $\widetilde{C}$ contains $\bar{C}$ or $\bar{C}$ is contained in $\widetilde{C}\left(\delta_{1}\right)$, where $\delta_{1}<\delta^{2} r 2^{r+1}$.

Proposition 14. - Let $C=b^{t} \stackrel{r}{\times} \bar{C}$ and let $\bar{C}$ be as in (G1), (G2), (G3) or (G4). Assume that

$$
d\left(C, \omega_{t}[l, l+|C|-1]\right)<\frac{\delta^{2}}{3}
$$

Then

$$
d\left(C^{\prime}, \omega_{t}\left[l^{\prime}, l^{\prime}+\left|C^{\prime}\right|-1\right]\right)<\delta^{2}
$$

where $C^{\prime}=b^{t} \stackrel{r}{\times} \widetilde{C}, \widetilde{C} \subset \omega_{t+1}$ and

(g1) $\widetilde{C}=b_{k^{\prime}}^{t+1}\left(i_{0}-1\right) \bar{C}_{2} b_{k^{\prime \prime}}^{t+1}\left(i_{1}+1\right), l^{\prime}=l-l_{1} r(c f$. (73), (74)), if (G1) holds,

(g2) $\widetilde{C}=b_{k^{\prime}}^{t+1}\left(i_{0}-1\right) \bar{C}_{2}, l^{\prime}=l-l_{1} r$, if (G2) holds,

(g3) $\widetilde{C}=\bar{C}_{2} b_{k^{\prime \prime}}^{t+1}\left(i_{1}+1\right), l^{\prime}=l$, if (G3) holds,

(g4) $\widetilde{C}=\bar{C}_{2}, l^{\prime}=l$, if (G4) holds.

Proof. - Consider the case (G2). Then (11) and (78) imply $\left(C_{2}=b^{t} \stackrel{r}{\times} \bar{C}_{2}\right)$

$$
d\left(b^{t} \stackrel{r}{\times} \overline{C_{2}}, \omega_{t}\left[\overline{l_{2}}, \overline{l_{2}}+\left|C_{2}\right|-1\right]\right)<\delta^{2}
$$

where $\overline{l_{2}}=l+\left|b^{t} \stackrel{r}{\times} \bar{C}_{1}\right|$.

It follows from Proposition 13 that $\omega_{t}\left[\overline{l_{2}}, \overline{l_{2}}+\left|C_{2}\right|-1\right]$ is of the form (75). Assume that the case (76) holds. Set

$$
\begin{gathered}
\widetilde{C_{1}}=\bar{C}_{1}\left[0,\left|\bar{C}_{1}\right|-j-1\right] \\
\widetilde{D_{1}}=\omega_{t+1}\left[\frac{1}{\lambda_{t}}(l-j), \frac{1}{\lambda_{t}}(l-j)+\left|\widetilde{C_{1}}\right|-1\right] \quad \text { (see Fig. 3). }
\end{gathered}
$$




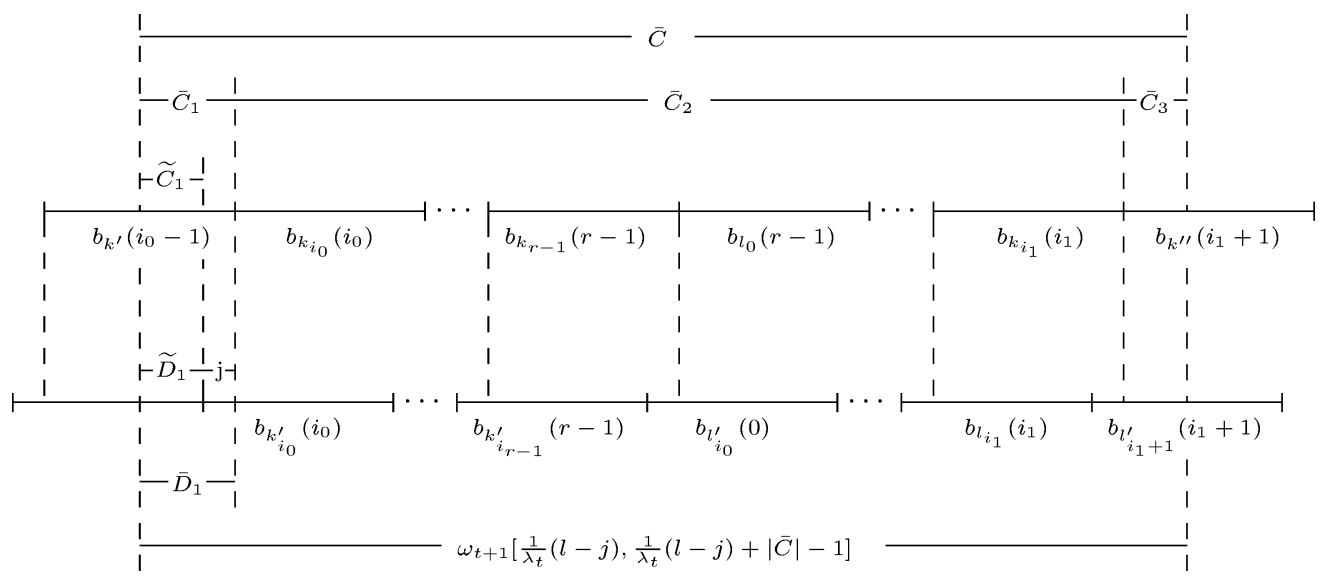

Fig. 3.

If follows from Proposition 8 that

$$
j \equiv\left(n-s_{0}\right) r 4(\bmod 4 n r)
$$

The fragment of $\omega_{t+1}$ from the left side of $b_{k_{0}^{\prime}}^{t+1}\left(i_{0}\right)$ having the length $\lambda_{t+1}$ is of a form $b_{u}^{t+1}\left(i_{0}-1\right)$ and either $u=k^{\prime}+s_{0}$ or $u=k^{\prime}+s_{0}+1$. Assume that $u=k^{\prime}+s_{0}+1$. Then Proposition 9 implies

$$
d\left(\widetilde{C_{1}}, \widetilde{D_{1}}\right)=1
$$

Let $\bar{D}_{1}$ denote the block $\omega_{t+1}\left[\frac{1}{\lambda_{t}}(l-j), \frac{1}{\lambda_{t}}(l-j)+\left|\bar{C}_{1}\right|-1\right]$ (see Fig. 3). Obviously we have

$$
\frac{\left|\bar{C}_{1}\right|}{|C|} d\left(\bar{C}_{1}, \bar{D}_{1}\right) \stackrel{(11),(8)}{\leqslant} d\left(C, \omega_{t}[l, l+|C|-1]\right)<\delta^{2}
$$

Further

$$
\begin{aligned}
& \delta^{2}>\frac{\left|\bar{C}_{1}\right|}{|\bar{C}|} d\left(\bar{C}_{1}, \bar{D}_{1}\right)>\delta d\left(\bar{C}_{1}, \bar{D}_{1}\right) \stackrel{(11))}{\geqslant} \frac{\left|\widetilde{C}_{1}\right|}{\left|\bar{C}_{1}\right|} \delta d\left(\widetilde{C_{1}}, \widetilde{D_{1}}\right) \stackrel{(80)}{=} \frac{\left|\bar{C}_{1}\right|-j}{\left|\bar{C}_{1}\right|} \delta \\
& =\delta\left(1-\frac{j}{\left|\bar{C}_{1}\right|}\right) \stackrel{(\mathrm{G} 2)}{\geqslant} \delta\left(1-\frac{j}{\delta|\bar{C}|}\right) \stackrel{(76)}{\geqslant} \delta\left(1-\frac{\delta^{2} r 2^{r+1} \lambda_{t+1}}{\delta|\bar{C}|}\right) \geqslant \delta\left(1-\delta r 2^{r+1}\right),
\end{aligned}
$$

because $|\bar{C}| \geqslant \lambda_{t+1}$.

Thus

$$
1-\delta r 2^{r+1}<\delta
$$

which is in contradiction with the inequality $\delta^{2}<1 /\left(r^{2} 2^{2 r+3}\right)$. We have shown $u-k^{\prime}=$ $s_{0}=k_{0}^{\prime}-k_{0}$.

Now, using (79) and the definition of $b_{k^{\prime}}\left(i_{0}-1\right)$ and $b_{u}\left(i_{0}-1\right)$ we obtain $C[v]=$ $\omega_{t}\left[l^{\prime}+v\right]$ for each $v=0, \ldots,\left|\bar{C}_{1}\right|-1, l^{\prime}=l-l_{1} r$ (see (74)). This last equality implies (g2). The proofs of the remaining cases are similar. 
Proposition 15. - Assume that $\mathcal{F}=\left\{C_{1}, \ldots, C_{d}\right\}, d \leqslant r-1$, is a family of subblocks of $\omega_{t}$ such that

$$
C_{j}=b^{t} \stackrel{r}{\times} \bar{C}_{j} \text { and each } \bar{C}_{j} \text { is as in (B). }
$$

Let $\omega_{t}(\mathcal{F})$ be the maximal subsequence of $\omega_{t}$ that can be $\delta^{2}$-covered by the family $\mathcal{F}$ in a disjoint way, $\delta^{2}<1 /\left(r^{2} 2^{2 r+3}\right)$, and let $\bar{\omega}_{t}(\mathcal{F})$ be the complementary part of $\omega_{t}$. Then it is an union of at least $(r-d)$ blocks $b^{t} \stackrel{r}{\times} b^{t+1}\left(i_{j}\right), j=1, \ldots, r-d$.

Proof. - Denote by $\mathcal{F}_{i}$ the set of all blocks $C \in \mathcal{F}$ such that $\bar{C} \delta^{2}$-covers a subblock of $\omega_{t+1}$ containing one of the form

$$
b_{1}^{t+1}(i) b^{t+1}(i+1), \quad i=0, \ldots, r-2,
$$

and by $\mathcal{F}_{r-1}$ those $C$ for which $\bar{C} \delta^{2}$-covers a block containing $b^{t+1}(r-1) b^{t+1}(0)$. We show that $\mathcal{F}_{i} \cap \mathcal{F}_{j}=\emptyset$ whenever $i \neq j$. Take $C \in \mathcal{F}_{i}, D \in \mathcal{F}_{j}$ and let $\bar{C}, \bar{D}$ be the blocks defined by (81), $\bar{C}$ as in (B) and

$$
\bar{D}=b_{i_{0}^{\prime}}^{t+1}\left(i_{0}^{\prime}\right) \ldots b_{k_{r-1}^{\prime}}^{t+1}(r-1) \mid b_{l_{0}^{\prime}}^{t+1}(0) \ldots b_{i_{1}^{\prime}}^{t+1}\left(i_{1}^{\prime}\right)
$$

If $\left(i_{0} \ldots(r-1) \mid 0 \ldots i_{1}\right) \neq\left(i_{0}^{\prime} \ldots(r-1) \mid 0 \ldots i_{1}^{\prime}\right)$ then $C \neq D$. If $\left(i_{0} \ldots(r-1) \mid\right.$ $\left.0 \ldots i_{1}\right)=\left(i_{0}^{\prime} \ldots(r-1) \mid 0 \ldots i_{1}^{\prime}\right)$ then using Proposition 13 we obtain

$$
\left(k_{i_{0}} \ldots k_{r-1} \mid l_{0} \ldots l_{i_{1}}\right)=\left(k_{i_{0}}^{\prime} \ldots k_{r-1}^{\prime} \mid l_{0}^{\prime} \ldots l_{i_{1}}^{\prime}\right)+s_{0}
$$

for some $s_{0} \in \mathbb{Z}_{n}$. The last condition is impossible since $i \neq j$. The proposition follows because $\#\left\{\mathcal{F}_{i} ; 0 \leqslant i<r\right\}=r$.

THEOREM 4. $-r\left(T_{\varphi}\right)=r$.

Proof. - According to Proposition 12 it remains to show that $r\left(T_{\varphi}\right)>r-1$. Let

$$
\frac{\delta^{2}}{9}<\frac{1}{r^{2} 2^{2 r+3}}
$$

and let $A_{1}, \ldots, A_{x}$ be blocks occurring in $\omega,\left|A_{i}\right| \geqslant p_{t_{0}}$ and $t_{0}$ satisfies $r / \lambda_{t}<\delta^{2} r 2^{r+1}$, if $t \geqslant t_{0}, x \leqslant r-1$. For each $u=1, \ldots, x$ there exists an unique $t=t(u)$ such that $A_{u}$ contains at least one $t$-symbol and does not contain any $(t+1)$-symbol. Then $A_{u}$ has a form

$$
A_{u}=\widetilde{E_{1}}\left(B^{t-1} \stackrel{r}{\times} C_{u}\right) \widetilde{E_{2}},
$$

where $C_{u} \subset \omega_{t}$ is as in $4.2,\left|C_{u}\right|=q r, q=q(u) \geqslant 1, E_{1}$ is a right-side part of a $t$-symbol and $E_{2}$ is a left-side part of a $t$-symbol. We divide the set $\{t(1), \ldots, t(x)\}$ by arithmetic order. More precisely, we put

$$
\tau_{1}=\max \{t(1), \ldots, t(x)\}, \quad T_{1}=\left\{u ; t(u)=\tau_{1}\right\}, \quad d_{1}=\# T_{1} .
$$


Next we define

$$
\tau_{2}=\max \left\{t(u) ; u \notin T_{1}\right\}, \quad T_{2}=\left\{u ; t(u)=\tau_{2}\right\}, \quad d_{2}=\# T_{2} .
$$

Similarly we define sets $T_{3}, \ldots, T_{v}$, numbers $\tau_{3}, \ldots, \tau_{v}$ and $d_{3}, \ldots, d_{v}$. We have

$$
\tau_{1}>\cdots>\tau_{v}, \quad d_{1}+\cdots+d_{v}=x .
$$

Let

$$
\mathcal{A}_{p}=\left\{A_{u} ; u \in T_{p}\right\}, \quad p=1, \ldots, v .
$$

The families $\mathcal{A}_{1}, \ldots, \mathcal{A}_{v}$ are pairwise disjoint and $\bigcup_{p=1}^{v} \mathcal{A}_{p}=\left\{A_{1}, \ldots, A_{x}\right\}$.

Consider the family $\mathcal{A}_{1}$. Assume that

$$
\mathcal{A}_{1}=\left\{A_{1}, \ldots, A_{d_{1}}\right\}
$$

Then

$$
C_{u}=b^{t} \stackrel{r}{\times} \bar{C}_{u}
$$

and

$$
\bar{C}_{u} \subset \omega_{t+1}, \quad u \in T_{1}, t=\tau_{1} .
$$

If $d\left(A_{u}, \omega\left[\widetilde{l}, \widetilde{l}+\left|A_{u}\right|-1\right]\right)<\delta^{2} / 9$ then by (11), (8),

$$
d\left(B^{t-1} \stackrel{r}{\times} C_{u}, \omega\left[l, l+m_{t-1}\left|C_{u}\right|-1\right]\right)<\frac{\delta^{2}}{3}
$$

where $l=\widetilde{l}+\left|\widetilde{E_{1}}\right|$.

According to Proposition 11

$$
d\left(C_{u}, \omega_{t}\left[l^{\prime}, l^{\prime}+\left|C_{u}\right|-1\right]\right)<\frac{\delta^{2}}{3}
$$

for some $l^{\prime} \in \mathbb{Z}$ and

$$
\left|l-p_{t} l^{\prime}\right|<\frac{1}{3} \delta^{2} r 2^{r+1} p_{t}
$$

We can write

$$
\bar{C}_{u}=\bar{C}_{u}^{(1)} \bar{C}_{u}^{(2)} \bar{C}_{u}{ }^{(3)}
$$

according to (73).

We distinguish among the blocks $A_{1}, \ldots, A_{d_{1}}$ three types $\mathcal{F}_{1}, \mathcal{F}_{2}, \mathcal{F}_{3}$, as follows;

$$
\begin{array}{ll}
A_{u} \in \mathcal{F}_{1} & \text { if } C_{u} \text { is as in (A) or (G4), } \\
A_{u} \in \mathcal{F}_{2} & \text { if } C_{u} \text { is as in (G1), (G2), or (G3), } \\
A_{u} \in \mathcal{F}_{3} & \text { if } C_{u} \text { is as in (B). }
\end{array}
$$


Let $d_{11}=\# \mathcal{F}_{1}, d_{12}=\# \mathcal{F}_{2}, d_{13}=\# \mathcal{F}_{3}$. We have

$$
d_{11}+d_{12}+d_{13}=d_{1} .
$$

Let $\omega\left(A_{1}, \ldots, A_{d_{1}}\right)$ be a subsequence of $\omega$ that is $\frac{\delta^{2}}{9}$-covered by the blocks $A_{1}, \ldots, A_{d_{1}}$ in a disjoint way. By $\omega\left(\mathcal{F}_{i}\right), i=1,2,3$, we denote the subsequence of $\omega \frac{\delta^{2}}{9}$-covered in a disjoint way by the families $\mathcal{F}_{i}$. Of course, $\omega\left(A_{1}, \ldots, A_{d_{1}}\right) \subset \omega\left(\mathcal{F}_{1}\right) \cup \omega\left(\mathcal{F}_{2}\right) \cup \omega\left(\mathcal{F}_{3}\right)$. Denoting by $\bar{\omega}\left(A_{1}, \ldots, A_{d_{1}}\right), \bar{\omega}\left(\mathcal{F}_{i}\right)$ the complementary parts of $\omega\left(A_{1}, \ldots, A_{d_{1}}\right), \omega\left(\mathcal{F}_{i}\right)$, $i=1,2,3$, respectively, we have

$$
\bar{\omega}\left(A_{1}, \ldots, A_{d_{1}}\right) \supset \bar{\omega}\left(\mathcal{F}_{1}\right) \cap \bar{\omega}\left(\mathcal{F}_{2}\right) \cap \bar{\omega}\left(\mathcal{F}_{3}\right) .
$$

According to (83)-(85) and Proposition 15 we have that $\bar{\omega}\left(\mathcal{F}_{3}\right)$ is an union of at least

$$
\left(r-d_{13}\right) \text { blocks } E\left(\delta_{1}\right),
$$

where

$$
\left\{\begin{array}{l}
E=B^{t} \stackrel{r}{\times} b^{t+1}\left(i_{j}\right), \quad j=1, \ldots, r-d_{13}, \quad \text { and } \\
\delta_{1} \leqslant 2 \delta^{2} r 2^{r+1}
\end{array}\right.
$$

because of

$$
\frac{\left|\widetilde{E_{1}}\right|}{\left|A_{u}\right|} \stackrel{(84)}{\leqslant} \frac{p_{t}}{m_{t+1}}=\frac{r}{\lambda_{t+1}}<\frac{1}{2} \delta_{1}, \quad \text { and } \quad \frac{\left|\widetilde{E_{2}}\right|}{\left|A_{u}\right|}<\frac{1}{2} \delta_{1} .
$$

Consider the family $\mathcal{F}_{2}$. Let $A_{u} \in \mathcal{F}_{2}$. If $A_{u} \frac{\delta^{2}}{9}$-covers a fragment $I_{u}$ of $\omega$ then (83) and (84) imply that $\overline{C_{u}} \frac{\delta^{2}}{3}$-covers a fragment $I_{u}=I_{u}(t)$ of $\omega_{t+1}$ and (85) implies

$$
I_{u} \subset\left(B^{t} \stackrel{r}{\times} I_{u}(t)\right)\left(\delta_{1}\right)
$$

It follows from Proposition 14 that there is $A_{\bar{u}}$ of a form as in $\mathcal{F}_{3}$ such that $\widetilde{C}_{\bar{u}} \frac{\delta^{2}}{3}$-covers another fragment $I_{\bar{u}}(t)$ of $\omega_{t+1}$ such that

$$
I_{u}(t) \subset I_{\bar{u}}(t)(\delta) .
$$

Applying Proposition 15 to the family $\left\{A_{\bar{u}}\right\}$ we obtain that $\bar{\omega}\left(\mathcal{F}_{3}\right) \cap \bar{\omega}\left(\mathcal{F}_{2}\right)$ is an union of at least $\left(r-d_{13}-d_{12}\right)$ blocks $E\left(\delta_{2}\right), E$ is as (87) and $\delta_{2}=\max \left(\delta, \delta_{1}\right)$.

Each block $E\left(\delta_{2}\right) \in \bar{\omega}\left(\mathcal{F}_{3}\right) \cap \bar{\omega}\left(\mathcal{F}_{2}\right)$ is an union of at least $\left(r-d_{13}-d_{12}\right)$ blocks of the form $B^{t} \stackrel{r}{\times} e_{k}^{(s)}, k \in \mathbb{Z}_{n}, s \in S, \# S=r-d_{13}-d_{12}$.

Using the same arguments as before we get that

$$
\left\{\begin{array}{l}
\bar{\omega}\left(\mathcal{F}_{3}\right) \cap \bar{\omega}\left(\mathcal{F}_{2}\right) \cap \bar{\omega}\left(\mathcal{F}_{1}\right) \text { is a union } \\
\text { of at least }\left(r-d_{13}-d_{12}-d_{11}\right) \text { blocks of the form } B^{t-1} \stackrel{r}{\times} e_{k}^{(s)}, \\
\quad s \in S_{1}, \# S_{1}=r-d_{13}-d_{12}-d_{11} .
\end{array}\right.
$$

Denoting $P\left(\omega_{1}, \omega\right)$ the density of a subsequence $\omega_{1}$ in $\omega$ and using (69), (86), (88) we have 


$$
\begin{aligned}
P\left(\bar{\omega}\left(A_{1}, \ldots, A_{d_{1}}\right), \omega\right) & \geqslant P\left(\bar{\omega}\left(\mathcal{F}_{3}\right) \cap \bar{\omega}\left(\mathcal{F}_{2}\right) \cap \bar{\omega}\left(\mathcal{F}_{1}\right), \omega\right) \\
& \geqslant\left(1-\frac{d_{13}+d_{12}}{r}\right)\left(1-\frac{d_{11}}{r}\right)\left(\frac{1}{4 n r}\right)^{2}\left(1-\delta_{2}\right) \\
& \geqslant\left(1-\frac{1}{r}\right)^{2}\left(\frac{1}{4 n r}\right)^{2}\left(1-\delta_{2}\right) \geqslant\left(1-\frac{1}{r}\right)^{2}\left(\frac{1}{4 n r}\right)^{2} \frac{1}{2} .
\end{aligned}
$$

If $T_{1} \neq\{1, \ldots, x\}$ then we repeat the above reasoning to the subsequence $\bar{\omega}\left(\mathcal{F}_{3}\right) \cap$ $\bar{\omega}\left(\mathcal{F}_{2}\right) \cap \bar{\omega}\left(\mathcal{F}_{1}\right)$ and $t=\tau_{2}$, and so on. As a consequence we get

$$
P\left(\bar{\omega}\left(A_{1}, \ldots, A_{x}\right), \omega\right) \geqslant\left(1-\frac{1}{r}\right)^{2 r} \frac{1}{2^{r}}\left(\frac{1}{4 n r}\right)^{2 r} .
$$

This implies $r\left(T_{\varphi}\right)>r-1$. Thus we have shown $r\left(T_{\varphi}\right)=r$.

\section{4. $r\left(T_{\varphi}\right)=r:$ the case 3.1}

To prove that $r\left(T_{\varphi}\right)=r$ in the case 3.1 we can repeat the same arguments an in 4.3. Similarly as in the Theorem 4 we consider blocks $A_{u}, u=1, \ldots, x, x \leqslant r-1$, and $A_{u}$ are as in (82), $C_{u}=b^{t} \stackrel{r}{\times} \bar{C}_{u}$ but $\bar{C}_{u}$ are as in (A), (B') and (G1), (G2), (G3), (G4).

As an analogue of Propositions 13-15 and Theorem 4 we obtain

Proposition 13'. - Assume that $C$ is as in $\left(\mathrm{B}^{\prime}\right)$ and let $d(C, D)<\delta^{2}, D \subset \omega_{t}$. Then $D$ has a form (75), and

$$
\bar{D}=b_{k_{i_{0}}^{\prime}}\left(i_{0}\right) . . b_{k_{r-1}^{\prime}}(r-1)\left|b_{u_{0}^{\prime}}(0) . . b_{u_{r-1}^{\prime}}(r-1)\right| b_{v_{0}}^{\prime}(0) . . b_{v_{r-1}^{\prime}}(r-1) \mid b_{l_{0}^{\prime}}(0) . . b_{l_{i_{1}}^{\prime}}\left(i_{1}\right)
$$

$b_{k}(i)=b_{k}^{t+1}(i)$, and $j$ satisfies either (76) or (77).

Proposition 14' ${ }^{\prime}$ - Let $C$ be as in Proposition $14, \bar{C}$ is as in (73) and $\bar{C}_{2}$ is as in $\left(\mathrm{B}^{\prime}\right)$. Then we get $(\mathrm{g} 1),(\mathrm{g} 2)$, (g3) or $(\mathrm{g} 4)$.

The proofs of Propositions $13^{\prime}$ and $14^{\prime}$ are similar to the proofs of Propositions 13 and 14.

Proposition $15^{\prime}$. - Let $\mathcal{F}=\left\{C_{1}, \ldots, C_{d}\right\}, d \leqslant r-1, C_{j}=b^{t} \stackrel{r}{\times} \bar{C}$, and $C_{j}$ are as in $\left(\mathrm{B}^{\prime}\right)$. Then we have the same thesis as in Proposition 15.

Proof. - Let $\mathcal{F}_{i, k}, i=0, \ldots, r-2, k \in \mathbb{Z}_{n}$, be the set of all blocks $C \in \mathcal{F}$ such that $\bar{C}\left(C=b^{t} \stackrel{r}{\times} \bar{C}\right) \delta^{2}$-covers a subblock of $\omega_{t+1}$ containing one of the form $b_{k}^{t+1}(i) b_{k+1}^{t+1}(i+$ 1). By $\mathcal{F}_{r-1, k}^{(1)}, \mathcal{F}_{r-1, k}^{(2)}$ we denote those $C \in \mathcal{F}$ such that $\bar{C}$ does so for the pairs $b_{k}^{t+1}(r-1) b_{k}^{t+1}(0)$ or $b_{k}^{t+1}(r-1) b_{k+1}^{t+1}(0)$ respectively. 
Using Propositions 3 and 7 we get that

$$
\left\{\begin{array}{l}
\text { if } \left.C \in \mathcal{F}_{i, k} \text { then } \bar{C} \delta^{2} \text {-covers (up to } \delta r^{2} 2^{r+3} \lambda_{t+1}\right) \text { only those } \\
\text { fragments of } \omega_{t+1} \text { containing blocks of the form } \\
\left(89^{\prime}\right) b^{t+1} \stackrel{r}{\times} \bar{e}_{k}^{(i+1)} \text { or } b^{t+1} \stackrel{r}{\times} \bar{e}_{k+4}^{(i)}, \text { if } n \geqslant 3, \\
\text { and } \\
\left(89^{\prime \prime}\right) b^{t+1} \stackrel{r}{\times} \bar{e}_{k}^{(i+1)} \text { if } n=2,
\end{array}\right.
$$

whenever $i=0, \ldots, r-2, k \in \mathbb{Z}_{n}$. Using Propositions 4 and 7 we get that

$$
\left\{\begin{array}{l}
\text { if } C \in \mathcal{F}_{r-1, k}^{(1)} \text { then } \bar{C} \delta^{2} \text {-covers only those fragments } \\
\text { of } \omega_{t+1} \text { containing blocks of the form } \\
\left(90^{\prime}\right) b_{k}^{t+1}(r-1) b_{k}^{t+1}(0) \text { or } b_{l}^{t+1}(r-1) b_{l+1}^{t+1}(0) \\
l \text { satisfies }(54)
\end{array}\right.
$$

and

$$
\left\{\begin{array}{l}
\text { if } C \in \mathcal{F}_{r-1, k}^{(2)} \text { then } \bar{C} \delta^{2} \text {-covers only those fragments } \\
\text { of } \omega_{t+1} \text { containing blocks of the form } \\
\left(91^{\prime}\right) b_{k}^{t+1}(r-1) b_{k+1}^{t+1}(0) \text { or } b_{l}^{t+1}(r-1) b_{l}^{t+1}(0) \\
l \text { satisfies }(54)
\end{array}\right.
$$

Now notice that each two blocks $b^{t+1} \stackrel{r}{\times} e_{k}^{(i)}$ and $b^{t+1} \stackrel{r}{\times} e_{k^{\prime}}^{(i)}, k^{\prime} \in \mathbb{Z}_{n}, k \neq k^{\prime}$, appearing in $\omega_{t+1}$ are separated by at least three blocks of the form $b^{t+1} \stackrel{r}{\times} e_{k+1}$. This, (89) and the condition $|E|<4 r\left(\right.$ see $\left.\left(\mathrm{B}^{\prime}\right)\right)$ imply that $\mathcal{F}_{i, k} \cap \mathcal{F}_{i, k^{\prime}}=\emptyset$, if $k \neq k^{\prime}, i=0, \ldots, r-2$. Similarly $\mathcal{F}_{r-1, k}^{(1)} \cap \mathcal{F}_{r-1, k^{\prime}}^{(1)}=\emptyset$ and $\mathcal{F}_{r-1, k}^{(2)} \cap \mathcal{F}_{r-1, k^{\prime}}^{(2)}=\emptyset$, if $k \neq k^{\prime}$.

Further (89) implies that if $C \in \mathcal{F}_{i, k} \cap \mathcal{F}_{i^{\prime}, k^{\prime}}$ then $i^{\prime}=i+1, k^{\prime}=k+4$ if $n \geqslant 3$ $\left(\left(89^{\prime}\right)\right)$ and $i^{\prime}=i, k^{\prime}=k$ if $n=2\left(\left(89^{\prime \prime}\right)\right), i=0, \ldots, r-2$. (90) implies that if $C \in$ $\mathcal{F}_{r-1, k}^{(1)} \cap \mathcal{F}_{r-1, k^{\prime}}^{(2)}$ then $k^{\prime}=l, l$ satisfying (54). Combining the above arguments we get that there is at least $\frac{r n}{2}-d$ fragments of $\omega_{t+1}$ of the form $\left(89^{\prime}\right)$ and (90) or (91) that are not covered by the family $\mathcal{F}$. The Proposition follows because $\frac{r n}{2} \geqslant r$.

THEOREM $4^{\prime} .-r\left(T_{\varphi}\right)=r$.

Proof. - We repeat the same reasoning as in the proof of Theorem 4 using blocks $A_{1}, \ldots, A_{x}$ of the form (82) with $q \geqslant 3$. We use Proposition 7 instead of Proposition 11 and the Propositions $14^{\prime}$ and 15' instead of Propositions 14 and 15. Then using (67) instead of (68) we get

$$
P\left(\bar{\omega}\left(A_{1}, \ldots, A_{x}\right), \omega\right) \geqslant\left(1-\frac{1}{r}\right)^{2 r} \frac{1}{2^{r}}\left(\frac{1}{r n 2^{r+1}}\right)^{2 r}
$$

what implies $r\left(T_{\varphi}\right)>r-1$ and by Proposition 12 we have $r\left(T_{\varphi}\right)=r$. 


\section{Pairs $(r, \infty)$ or $(\infty, m)$}

In this part we construct group extensions $\left(X \times G, T_{\varphi}\right)$ such that $r\left(T_{\varphi}\right)=r, q\left(T_{\varphi}\right)=$ $\infty, 2 \leqslant r<\infty$ or $r\left(T_{\varphi}\right)=\infty, q\left(T_{\varphi}\right)=m, 1 \leqslant m<\infty$.

\subsection{The case $(r, \infty)$}

Take a sequence $\left\{s_{t}\right\}_{t=0}^{\infty}, s_{t+1}=\mu_{t+1} s_{t}, s_{0}=\mu_{0}, \mu_{t} \geqslant 2$ for $t \geqslant 0$ and let $G$ be the group of $\left\{s_{t}\right\}$-adic integers. Let $e=1+0 s_{1}+0 p_{2}+\cdots$. The set of all $\left\{s_{t}\right\}$-adic rational integers of $G$ coincides with the set $\left\{e_{n}, n \in Z\right\}$, where $e_{n}=n e$. Similarly as in the case 3.1 we define an adding machine $(X, \mathcal{B}, \mu, T)$ and a cocycle $\varphi: X \longrightarrow G$. To do this we define blocks $F^{(0)}, F^{(1)}, \ldots, F^{(r-1)}(r \geqslant 2$ is given) over $G$.

Put

$$
\begin{aligned}
F^{(i)}(t) & =F^{(i)}=\overbrace{0 \ldots 0}^{r\left(2^{i+1}-1\right)} \overbrace{0 \ldots 0 e 0 \ldots 0}^{r}, \quad i=0, \ldots, r-1, \\
H^{(i)} & =F^{(i)} F_{e}^{(i)} \ldots F_{\left(s_{t}-1\right) e}^{(i)} .
\end{aligned}
$$

Then $\left|H^{(i)}\right|=s_{t} r 2^{t+1}$. Next define $b^{t}(0), \ldots, b^{t}(r-1)$ as in 3.1 and $b^{t}=b^{t}(0) \ldots b^{t}(r-$ $1), t \geqslant 0$.

We have

$$
\lambda_{t}=\left|b^{t}(i)\right|=s_{t} r 2^{r+t+1}, \quad i=0, \ldots, r-1
$$

and

$$
\left|b^{t}\right|=s_{t} r^{2} 2^{r+t+1} \text {. }
$$

Then we define the blocks $B^{t}, t \geqslant 0$, by (14). We have $p_{t}=\left|B^{t}\right|=s_{0} \ldots s_{t} r^{2 t} 2^{r+1}\left(2^{t+1}\right.$ $1)$. Let $(X, \mathcal{B}, \mu, T)$ be the $\left\{p_{t}\right\}$-adic adding machine and define a cocycle $\varphi: X \longrightarrow G$ by (18).

THEOREM 5. $-r(T \varphi)=r$ and $q\left(T_{\varphi}\right)=\infty$

Proof. - Let $\Pi_{t}: G \longrightarrow Z / s_{t} Z$ be the natural group homomophism. We can define cocycles $\varphi_{t}: X \longrightarrow Z / s_{t} Z$ by $\varphi_{t}=\varphi \circ \Pi_{t}$. It is evident that $\varphi_{t}$ is a $r$-Toeplitz cocycle as in 3.1 defined by the blocks $\Pi_{t}\left(B_{k}\right), u \geqslant 0$. According to Theorems 2 and 4 we have $r\left(T_{\varphi_{t}}\right)=r$ and $q\left(T_{\varphi_{t}}\right)=s_{t}$. It follows from the definitions of $\varphi$ and $\varphi_{t}$ that the dynamical system $\left(X \times G, T_{\varphi}\right)$ is the inverse limit of the systems $\left(X \times Z / s_{t} Z, T_{\varphi_{t}}\right)$. Then from the definition of the rank we obtain $r\left(T_{\varphi}\right)=r$. It is proved in Theorem 2 that $\sigma_{j e} \notin \operatorname{wcl}\left\{T_{\varphi_{t}}^{n}, n \in Z\right\}$ if $j=0, \ldots, s_{t}-1, t \geqslant 0$. This means that $\sigma_{j e} \notin \operatorname{wcl}\left\{T_{\varphi}^{n}, n \in Z\right\}$ for every $j \in Z, j \neq 0$ which implies $q\left(T_{\varphi}\right)=\infty$.

\subsection{The case $(\infty, m)$}

First consider the case $m \geqslant 2$. Let $r_{t}=2^{t+1}, t \geqslant 0$, and define blocks $F^{(i)}=F^{(i)}(t)$ over $G=Z / m Z, i=0, \ldots, r_{r+1}-1$, as follows:

$$
v F^{(i)}=\overbrace{0 \ldots 0}^{2^{i+1} r_{t}} \overbrace{0 \ldots 0 \underbrace{1}_{i+1} 0 \ldots 0}^{r_{t+1}} 0,
$$




$$
H^{(i)}=F_{0}^{(i)} F_{1}^{(i)} \ldots F_{m-1}^{(i)}, \quad i=0, \ldots, r_{t+1}-1 .
$$

We have $\left|H^{(i)}\right|=m r_{t} 2^{i+3}$. Next define $b^{t}(0), \ldots, b^{t}\left(r_{t+1}-1\right), b^{t}, B^{t}$ by putting

$$
\begin{aligned}
b^{t}(i) & =\overbrace{H^{(i)} H^{(i)} \ldots H^{(i)}}^{x}, \quad x=2^{t+r_{t+1}-i-1} \\
b^{t} & =b^{t}(0) b^{t}(1) \ldots b^{t}\left(r_{t+1}-1\right), \quad \text { and } \\
B^{t} & =b^{0} \stackrel{r_{0}}{\times} b^{1} \stackrel{r_{1}}{\times} \ldots \stackrel{r_{i-1}}{\times} b^{t} .
\end{aligned}
$$

Then $\lambda_{t}=\left|b^{t}(i)\right|=m 2^{2 t+\rho+2}, \rho=r_{t+1}$ and $p_{t}=m_{t} r_{t+1}, m_{t}=\lambda_{0} \cdots \lambda_{t}$. We define a cocycle $\varphi: X \longrightarrow G$ by

$$
\varphi(x)=B^{t}[j+1]-b^{t}[j]
$$

if $x \in D_{j}^{t}$ except if $j=m_{t}-1, \ldots, p_{t}-1$. The cocycle $\varphi$ is constant on the levels $D_{j}^{t}$ except of $r_{t+1}$ consecutive levels.

In a similar way we construct a cocycle $\varphi$ if $m=1$. Take $n$ as in the case 3.2 and define

$$
\begin{aligned}
F^{(i)}(t) & =F^{(i)}=\overbrace{0 \ldots 0}^{2 r_{t}} \overbrace{0 \ldots 0 \underbrace{1}_{i+1} 0 \ldots 0}^{2 r_{t}}, \\
H^{(i)} & =F_{0}^{(i)} F_{1}^{(i)} \ldots F_{n-1}^{(i)}, \quad i=0,1, \ldots, r_{t+1}-1 .
\end{aligned}
$$

The next steps of the definition $\varphi$ are the same as in the case $m \geqslant 2$.

THEOREM 6. $-r\left(T_{\varphi}\right)=\infty, q\left(T_{\varphi}\right)=m$ and $\operatorname{wcl}\left\{T_{\varphi}^{n}, n \in z\right\}$ is uncountable.

Proof. - For the dynamical system $\left(X \times G, T_{\varphi}\right)$ we can use the same arguments as in the parts 3 and 4 taking $r_{t}$ instead of $r$. Theorems 2, 3 and $3^{\prime}$ are valid. To estimate the rank of $T_{\varphi}$ we use the shift representations $\left(\Omega \omega, T_{\sigma}\right)$ of $\left(X \times G, T_{\varphi}\right)$ where $\omega=b^{0} \stackrel{r_{0}}{\times} b^{1} \stackrel{r_{1}}{\times} \ldots$. Repeating the proof of Theorem 4 and $4^{\prime}$ we get $r\left(T_{\varphi}\right)>r_{t}-1$ for every $t \geqslant 0$. Thus $r\left(T_{\varphi}\right)=\infty$.

\section{The pair $(\infty, \infty)$}

This case is easy to handle: first let $(Y, S, v)$ be the rank 1 mixing staircase transformation [1]. Then let $G$ denote the group of dyadic integers, and let $m_{G}$ denote its normalized Haar measure.

Then consider a Morse cocycle [23] $\phi: Y \rightarrow G$, that is a measurable map which is constant on the levels of the stacks defining the rank $1 \mathrm{~S}$, except the top level, at each step.

To select a $\phi$ such that the system $\left(Y \times G, S_{\phi}, v \otimes m_{G}\right)$ is mixing, where

$$
S_{\phi}(y, g)=(S y, g+\phi(y)),
$$

we proceed as follows. 
The system is the inverse limit of the sequence of systems $\left(Y \times Z / 2^{t} Z, S_{\phi_{t}}, v \otimes m_{t}\right)$ where $m_{t}$ is Haar measure on $Z / 2^{t} Z$ and $\phi_{t}=\pi_{t} \circ \phi$.

Therefore [13] enough is to make sure that $\phi$ is such that each $S_{\phi_{t}}$ is mixing. Using [24], sufficient is that each $\phi_{t}$ is a weakly-mixing cocycle. This in turn is easy to ensure using [14, Theorems 3, 4].

So we take a $\phi$ such that $S_{\phi}$ is mixing. Now because $S_{\phi_{t}}$ is a factor of $S_{\phi}$, we have the inequality $r\left(S_{\phi_{t}}\right) \leqslant r\left(S_{\phi}\right)$.

But since $S_{\phi_{t}}$ is mixing, using [12], it follows that $r\left(S_{\phi_{t}}\right)=2^{t}$. Whence $r\left(S_{\phi}\right)=\infty$.

Now $S_{\phi}$ is mixing therefore $\left\{S_{\phi}^{n}: n \in Z\right\}=\operatorname{wcl}\left\{S_{\phi}^{n}: n \in Z\right\}$. Else for each $g \in G$, $\sigma_{g} \in C\left(S_{\phi}\right)$, and $G$ is uncountable.

We deduce that $q\left(S_{\phi}\right)=\infty$.

\section{REFERENCES}

[1] Adams T., Smorodinsky's conjecture on rank 1 mixing, Proc. Amer. Math. Soc. 126 (1998) 739-744.

[2] Christol G., Kamae T., Mendès France M., Rauzy G., Suites algébriques, automates et substitutions, Bull. Soc. Math. France 108 (1980) 401-419.

[3] del Junco A., A transformation with simple spectrum which is not rank one, Canad. J. Math. 29 (1977) 655-663.

[4] Ferenczi S., Systèmes localement de rang un, Ann. Inst. Henri Poincaré, Probab. Stat. 20 (1984) 35-51.

[5] Ferenczi S., Systèmes de rang un gauche, Ann. Inst. Henri Poincaré, Probab. Stat. 21 (1985) $177-186$.

[6] Ferenczi S., Systems of finite rank, Colloq. Math. 73 (1) (1997) 35-65.

[7] Ferenczi S., Kwiatkowski J., Rank and spectral multiplicity, Stud. Math. 102 (2) (1992) $121-144$.

[8] Filipowicz I., Kwiatkowski J., Rank, covering number and simple spectrum, J. d'Analyse Math. 66 (1995) 185-216.

[9] Goodson G., Kwiatkowski J., Liardet P., Lemaǹczyk M., On the multiplicity function of ergodic group extensions of rotations, Studia Math. 102 (1992) 157-174.

[10] King J., The commutant is the weak closure of the powers, for rank-1 transformations, Ergodic Theory Dynam. Systems 6 (1986) 363-385.

[11] King J., Joining - rank and the structure of finite rank mixing transformations, J. d'Analyse Math. 51 (1988) 182-227.

[12] King J., A lower bound for the rank of mixing transformations, Israel J. Math. 59 (1987) 377-380.

[13] Kwiatkowski J., Inverse limit of $M$-cocycles and applications, Funda. Math. 157 (1998) 261-276.

[14] Kwiatkowski J., Lacroix Y., Morse extensions of rank 1 systems, Prepint, U.B.O. No 041998, http://gauss.univ-brest.fr/recherche/prepub/1998.html.

[15] Kwiatkowski J., Lemańczyk M, On the multiplicity function of ergodic group extensions, Studia Math. 116 (1995) 207-215.

[16] Lemańczyk M., Toeplitz $\mathbb{Z}_{2}$-extensions, Ann. Inst. Henri Poincaré 24 (1988) 1-43.

[17] Lemańczyk M., Liardet P., Coalescence of Anzai skew product, unpublished preprint.

[18] Lemańczyk M., Liardet P., Thouvenot J.P., Coalescence of circle extensions of measurepreserving transformations, Ergodic Theory Dynam. Systems 12 (1992) 769-789. 
[19] Lemańczyk M., Mentzen M., Compact subgroups in the centralizer of natural factors of an ergodic group extension of a rotation determine all factors, Ergodic Theory Dynam. Systems 10 (1990) 763-776.

[20] Mentzen M., Thesis, Preprint no 2/89, Nicholas Copernicus University, Toruń (1989).

[21] Newton D., On canonical factors of ergodic dynamical systems, J. London Math. Soc. 219 (1979) 129-136.

[22] Parry W., Compact abelian group extensions of discrete dynamical systems, Z. Wahr. Verv. Geb. 13 (1969) 95-113.

[23] Rojek T., On metric isomorphism of Morse dynamical systems, Studia Math. 84 (1986) 247-267.

[24] Rudolph D.J., $K$-fold mixing lifts to weakly mixing isometric extensions, Ergodic Theory Dynam. Systems 5 (1985) 445-447. 